\title{
DERIVED CATEGORIES OF THADDEUS PAIR MODULI SPACES VIA D-CRITICAL FLIPS
}

\author{
NAOKI KOSEKI AND YUKINOBU TODA
}

\begin{abstract}
We show that the moduli spaces of Thaddeus pairs on smooth projective curves and those of dual pairs are related by d-critical flips, which are virtual birational transformations introduced by the second author. We then prove the existence of fully-faithful functors between derived categories of coherent sheaves on these moduli spaces. Our result gives an evidence of a d-critical analogue of Bondal-Orlov, Kawamata's D/K equivalence conjecture, and also a categorification of wall-crossing formula of Donaldson-Thomas type invariants on ADHM sheaves introduced by Diaconescu.
\end{abstract}

\section{Contents}

1. Introduction 1

2. Derived factorization categories under variation of GIT quotients 5

3. Derived categories of representations of quivers 14

4. Geometry of moduli spaces of Thaddeus pairs 21

5. Derived functors between Thaddeus pair moduli spaces 31

6. Comparison with ADHM sheaves 42

Appendix A. Review on d-critical birational geometry 45

References 47

\section{INTRODUCTION}

The purpose of this paper is to show the existence of fully-faithful functors between derived categories of coherent sheaves on moduli spaces of Thaddeus pairs on smooth projective curves and those of dual Thaddeus pairs. They are smooth projective varieties, and higher rank generalizations of symmetric products of curves. In this introduction, we first state the main result of this paper, then discuss the motivation of our result from the view point of d-critical birational geometry, and finally give an outline of the proof of the main theorem.

1.1. Main result. Let $C$ be a smooth projective curve over $\mathbb{C}$ with genus g. By definition, a Thadeus pair on $C$ is a pair Tha94]

$$
(E, s), s \in H^{0}(C, E)
$$


where $E$ is a semistable vector bundle on $C$, satisfying the following stability condition: there is no non-zero subbundle $F \subsetneq E$ satisfying $\mu(F)=\mu(E)$ and $s \in H^{0}(C, F)$, where $\mu(F):=\chi(F) / \operatorname{rank}(F)$.

We denote by

$$
M^{\mathrm{T}}(r, d)
$$

the moduli space of Thaddeus pairs (1.1), such that the bundle $E$ satisfies the numerical condition

$$
(\operatorname{rank}(E), \chi(E))=(r, d) .
$$

The moduli space $M^{\mathrm{T}}(r, d)$ is a smooth projective variety with dimension $d+r^{2}(g-1)$. We consider the following diagram

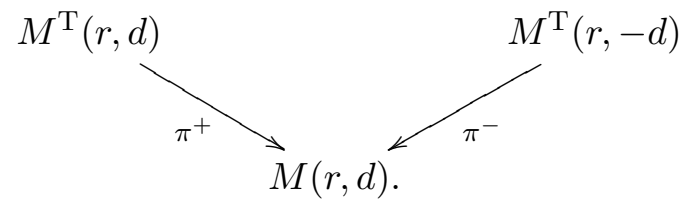

Here $M(r, d)$ is the coarse moduli space of $S$-equivalence classes of semistable bundles on $C$ satisfying the condition (1.2), and the morphisms $\pi^{ \pm}$are defined by

$$
\pi^{+}(E, s)=[E], \pi^{-}\left(E^{\prime}, s^{\prime}\right)=\left[E^{\prime \vee} \otimes \omega_{C}\right] .
$$

Theorem 1.1. (Theorem 4.10, Theorem 5.2) Suppose that $d \geq 0$.

(i) The diagram (1.3) is a d-critical flip for $d>0$, a $d$-critical flop for $d=0$.

(ii) We have a fully-faithful functor

$$
\Phi_{M}: D^{b}\left(M^{\mathrm{T}}(r,-d)\right) \hookrightarrow D^{b}\left(M^{\mathrm{T}}(r, d)\right) .
$$

Here both sides are derived categories of coherent sheaves, and the functor $\Phi_{M}$ is an equivalence for $d=0$.

Here we refer to Definition A.4 for the notion of d-critical flips, flops. It is natural to interpret the moduli space $M^{\mathrm{T}}(r,-d)$ in terms of dual Thaddeus pairs, i.e. it is the moduli space of pairs

$$
\left(E^{\prime \prime}, s^{\prime \prime}\right), s^{\prime \prime} \in \operatorname{Hom}\left(E^{\prime \prime}, \omega_{C}\right)
$$

where $E^{\prime \prime}$ is a semistable bundle on $C$ satisfying the condition (1.2), and a stability condition similar to the pair (1.1) (see Lemma 4.3).

In Theorem 5.10, we will also show that the fully-faithful functor $\Phi_{M}$ restricted to the stable part $M^{\text {st }}(r, d) \subset M(r, d)$ is given by a Fourier-Mukai functor whose kernel is a line bundle on the fiber product of the diagram (1.3) over $M^{\text {st }}(r, d)$. Therefore for $d=0$, the functor $\Phi_{M}$ gives a non-trivial autequivalence of $D^{b}\left(M^{\mathrm{T}}(r, 0)\right)$. We also remark that for $r=1$, we have

$$
M^{\mathrm{T}}(1, d)=S^{g+d-1}(C)
$$

and Theorem 1.1 in this case is a corollary of the main result of [Todb]. 
1.2. Motivation and Background. In [Toda], the second author introduced the notion of d-critical flips, flops, for diagrams of Joyce's d-critical loci Joy15, as an analogy of usual flips, flops, in birational geometry. In general they are not birational in the usual sense, and should be interpreted as virtual birational maps. Such a d-critical birational transformation typically occurs when we consider wall-crossing diagram of moduli spaces of stable objects on Calabi-Yau 3-folds.

On the other hand if two smooth varieties are related by a flip (flop) in the usual sense, then Bondal-Orlov [BO] and Kawamata Kaw02 conjectured the existence of a fully-faithful functor (equivalence) of their derived categories of coherent sheaves. This conjecture is called a $D / K$ conjecture. We expect an analogy of $\mathrm{D} / \mathrm{K}$ conjecture for $\mathrm{d}$-critical loci, i.e. under a d-critical flip (flop), there may exist categorifications of Donaldson-Thomas theory which are related by a fully-faithful functor (equivalence).

In [Toda], it turned out that wall-crossing diagrams of PandharipandeThomas stable pair moduli spaces on Calabi-Yau 3-folds [PT09, used in showing the rationality of their generating series [Tod10, Tod12, form a d-critical minimal model program. Based on this observation, we expected existence of fully-faithful functors of certain categorifications of DonaldsonThomas theory under the above wall-crossing (see [Todb, Section 1]). If this is true, then it gives a link between categorifications of wall-crossing formula of Donaldson-Thomas theory and a d-critical analogue of $\mathrm{D} / \mathrm{K}$ equivalence conjecture.

In the previous paper [Todb], the second author studied the above expectation in the case of wall-crossing of stable pair moduli spaces on Calabi-Yau 3 -folds, under the assumption that the curve class is irreducible and the relevant moduli spaces are non-singular. More precisely in [Todb], we proved the existence of fully-faithful functors of derived categories of coherent sheaves on stable pair moduli spaces in a wall-crossing diagram similar to (1.3), under the assumption mentioned above. In this case the wall-crossing diagram is a simple d-critical flip, and the situation is much easier than the diagram (1.3). However if the curve class is not irreducible, then the wall-crossing diagram is no more simple, and we cannot apply the above strategy used in Todb.

The result of Theorem 1.1 is motivated by extending the result of Todb for non-irreducible curve classes. Indeed we will see in Section 6 that the diagram (1.3) is a $\mathbb{C}^{*}$-fixed locus of a wall-crossing diagram which appeared in [Tod10], for a non-compact Calabi-Yau 3-fold $X$ of the form

$$
X=\operatorname{Tot}_{C}\left(M_{1}^{\vee} \oplus M_{2}^{\vee}\right), M_{1} \otimes M_{2} \cong \omega_{C}^{\vee} .
$$

Here $M_{1}, M_{2}$ are line bundles on $C$. The relevant stable objects on the above $X$ are described in terms of ADHM sheaves introduced by Diaconescu Dia12a, Dia12b. The rank $r$ of our vector bundle corresponds to the curve class $r[C]$ where $[C]$ is the class of the zero section of $X \rightarrow C$. Thus for $r \geq 2$, the result of Theorem 1.1 gives a certain extension of 
the main result of [Todb] for non-irreducible (indeed non-primitive) curve classes, when $X$ is of the form (1.4).

1.3. Strategy of the proof of Theorem 1.1 (ii). In Todb, we constructed a fully-faithful functor of derived categories of stable pair moduli spaces by the following steps: we first constructed a fully-faithful functor locally on the base, then described the kernel object explicitly, and finally used the description of the kernel object to construct a global fully-faithful functor. In our situation the diagram (1.3) is not a simple d-critical flip (flop), and it is much harder to employ the above strategy, e.g. the description of the kernel object is difficult. Instead we borrow an idea from HLa, using window subcategories developed in [HL15, BFK19], magic window theorem [vVdB17, HLS], together with analytic local description of the diagram (1.3) proved in [Tod18.

Here we give an outline of the proof of Theorem 1.1 (ii). Let $\mathcal{M}(r, d)$ be the moduli stack of semistable bundles $E$ on $C$ satisfying the condition (4.1), and $\mathcal{E} \rightarrow C \times \mathcal{M}(r, d)$ the universal bundle. We write $\operatorname{Rpr}_{\mathcal{M} *} \mathcal{E}$ as a two term complex of vector bundles

$$
\operatorname{Rpr}_{\mathcal{M} *} \mathcal{E}=\left(\mathcal{F}_{0} \stackrel{\psi}{\rightarrow} \mathcal{F}_{1}\right)
$$

where $\operatorname{pr}_{\mathcal{M}}: C \times \mathcal{M}(r, d) \rightarrow \mathcal{M}(r, d)$ is the projection. We then define

$$
w: \mathcal{Y}:=\mathcal{F}_{0} \times_{\mathcal{M}(r, d)} \mathcal{F}_{1}^{\vee} \rightarrow \mathbb{A}^{1}
$$

where $w$ is naturally defined using the map $\psi$. We show that there exist open immersions

$$
M^{\mathrm{T}}(r, \pm d) \subset \mathcal{W}:=\{d w=0\}
$$

which are realized as GIT semistable loci with respect to some $\mathbb{Q}$-line bundles $L^{ \pm}$on $\mathcal{Y}$ restricted to $\mathcal{W}$.

We then use the window theorem [HL15, BFK19] for the $\mathbb{C}^{*}$-equivariant derived factorization category $D_{\mathbb{C}^{*}}(\mathcal{Y}, w)$, and construct subcategories together with equivalences

$$
\mathcal{C}_{\mathcal{Y}}^{ \pm} \subset D_{\mathbb{C}^{*}}(\mathcal{Y}, w), \mathcal{C}_{\mathcal{Y}}^{ \pm} \stackrel{\sim}{\rightarrow} D_{\mathbb{C}^{*}}\left(\mathcal{Y}_{L^{ \pm-s s}}, w\right)
$$

Moreover by (1.5), a version of Knörrer periodicity Isi13, Shi12, Hir17] implies the equivalences

$$
D^{b}\left(M^{\mathrm{T}}(r, \pm d)\right) \stackrel{\sim}{\rightarrow} D_{\mathbb{C}^{*}}\left(\mathcal{Y}_{L^{ \pm}-\mathrm{ss}}, w\right) .
$$

We are reduced to showing the inclusion $\mathcal{C}_{\mathcal{Y}}^{-} \subset \mathcal{C}_{\mathcal{Y}}^{+}$, which is now a local statement on $M(r, d)$. The result of [Tod18] shows that the diagram (1.3) is analytic locally on $M(r, d)$ described as moduli spaces of representations of a quiver $Q^{\dagger}$ with a super-potential. The quiver $Q^{\dagger}$ is not necessary symmetric, but we can find its subquiver $Q_{0}^{\dagger}$ which is symmetric, thus its representation space is a symmetric representation of a reductive group. Then applying the magic window theorem $\mathrm{vVdB17}$, HLS for quasi-symmetric representations of reductive groups, we conclude the inclusion $\mathcal{C}_{\mathcal{Y}}^{-} \subset \mathcal{C}_{\mathcal{Y}}^{+}$. 
1.4. Related works. This paper is regarded as a sequel of the second author's previous paper [Todb, where we proved the existence of a fullyfaithful functor between (simpler) d-critical flips of stable pair moduli spaces.

In Pot16, it is proved that under wall-crossing of pair moduli spaces $\left(\mathcal{O}_{C} \rightarrow E\right)$ there exist fully-faithful functors of derived categories. The moduil spaces of stable pairs considered in loc. cit. are birational under wall-crossing, and different from the wall-crossing considered here. In terms of $\delta$-stability of ADHM sheaves (see Remark 6.3), the wall-crossing in [Pot16] considers walls located in $\delta>0$, while our wall-crossing corresponds to the wall at $\delta=0$.

There exist some other works proving the existence of fully-faithful functors between derived categories of stable objects. In [Bal17], Ballard proved the existence of full-faithful functor for wall-crossing of stable sheaves on some rational surfaces. In [HLa], Halpern-Leistner announces the result that the derived categories of stable objects on K3 surfaces are equivalent under wall-crossing. In Bal17, HLa, they use window subcategories to show their results. The moduli spaces in loc. cit. are birational under wall-crossing, so the situation is different from ours. However we are much influenced by their works, and borrowed several ideas from them.

1.5. Acknowledgements. We are grateful to Yuki Hirano and Daniel HalpernLeistner for valuable discussions. N. K. is supported by the program for Leading Graduate Schools, MEXT, Japan, and by Grant-in-Aid for JSPS Research Fellow 17J00664. Y. T. is supported by World Premier International Research Center Initiative (WPI initiative), MEXT, Japan, and Grant-in Aid for Scientific Research grant (No. 26287002) from MEXT, Japan.

1.6. Notation and convention. In this paper, all the schemes and stacks are defined over $\mathbb{C}$. For a scheme or a stack $M$, we always denote by $D^{b}(M)$ the bounded derived category of coherent sheaves on $M$.

\section{Derived FaCtorization CATEgories Under VARIATION OF GIT QUOTIENTS}

In this section, we recall some necessary background on derived factorization categories and their window subcategories. We will also show some inclusion of window subcategories as an application of magic window theorem $\mathrm{vVdB17}$, HLS].

2.1. Kempf-Ness stratification. Let $G$ be a reductive algebraic group, with maximal torus $T \subset G$. We always denote by $M$ the character lattice of $T$ and $N$ the cocharacter lattice of $T$, i.e.

$$
M=\operatorname{Hom}_{\mathbb{Z}}\left(T, \mathbb{C}^{*}\right), N=\operatorname{Hom}_{\mathbb{Z}}\left(\mathbb{C}^{*}, T\right) .
$$

The subspace $M_{\mathbb{R}}^{W} \subset M_{\mathbb{R}}$ is defined to be the Weyl-invariant subspace. 
Below we follow the convention of [HL15, Section 2.1] for Kempf-Ness stratification associated with GIT quotients. Let $X$ be a smooth variety, projective over an affine variety, with a $G$-action. For a $G$-linearized ample line bundle $L$ on $X$, we have the open subset of $L$-semistable points

$$
X_{L \text {-ss }} \subset X \text {. }
$$

By the Hilbert-Mumford criterion, $X_{L \text {-ss }}$ is characterized by the set of points $x \in X$ such that for any one parameter subgroup $\lambda: \mathbb{C}^{*} \rightarrow G$ for which the limit

$$
y=\lim _{t \rightarrow 0} \lambda(t)(x) \in X
$$

exists, we have weight ${ }_{\lambda}\left(\left.L\right|_{y}\right) \geq 0$. Also by fixing a Weyl-invariant norm $|*|$ on $N_{\mathbb{R}}$, we have the associated Kempf-Ness (KN) stratification

$$
X=X_{L \text {-ss }} \sqcup S_{1} \sqcup S_{2} \sqcup \ldots
$$

Here for each $\alpha$ there exists a one parameter subgroup $\lambda_{\alpha}: \mathbb{C}^{*} \rightarrow T$, a connected component (called center) $Z_{\alpha}$ of the $\lambda_{\alpha}$-fixed part of $X \backslash \cup_{\alpha^{\prime}<\alpha} S_{\alpha}$ such that

$$
S_{\alpha}=G \cdot Y_{\alpha}, Y_{\alpha}:=\left\{x \in X: \lim _{t \rightarrow 0} \lambda_{\alpha}(t)(x) \in Z_{\alpha}\right\} .
$$

Moreover by setting

$$
\mu_{\alpha}=-\frac{\text { weight }_{\lambda_{\alpha}}\left(\left.L\right|_{Z_{\alpha}}\right)}{\left|\lambda_{\alpha}\right|} \in \mathbb{R}
$$

we have the inequalities $\mu_{1}>\mu_{2}>\cdots>0$.

By taking the quotient stacks of the stratification (2.1), we have the stratification of the quotient stack $\mathcal{X}=[X / G]$

$$
\mathcal{X}=\mathcal{X}_{L \text {-ss }} \sqcup \mathcal{S}_{1} \sqcup \mathcal{S}_{2} \sqcup \ldots
$$

Remark 2.1. The stratification (2.2) is the $\Theta$-stratification introduced in [HLb], which is intrinsic to the stack $\mathcal{X}$, uniquely determined by $L \in \operatorname{Pic}(\mathcal{X})$ and some $b \in H^{4}(B G)$ pulled back via $\mathcal{X} \rightarrow B G$ (see [HLb, Example 4.13]).

2.2. Derived factorization categories. Suppose that there exists a subtorus $\mathbb{C}^{*} \subset G$ which is contained in the center of $G$. We set $\mathbb{P}(G):=G / \mathbb{C}^{*}$ and define

$$
\widetilde{G}:=G \times \times_{\mathbb{P}(G)} G .
$$

Here $G \rightarrow \mathbb{P}(G)$ is the natural quotient map. We have the exact sequence of algebraic groups

$$
1 \rightarrow G \stackrel{\Delta}{\rightarrow} \widetilde{G} \stackrel{\tau}{\rightarrow} \mathbb{C}^{*} \rightarrow 1 .
$$

Here $\Delta$ is the diagonal embedding, and $\tau$ sends $\left(g_{1}, g_{2}\right)$ to $g_{1} g_{2}^{-1}$. Below we regard $G$ as a subgroup of $\widetilde{G}$ by the diagonal $\Delta$, and whenever we have a $\widetilde{G}$-action we also regard it as a $G$-action by the embedding $\Delta$. 
The exact sequence (2.4) splits non-canonically, i.e. for any $k \in \mathbb{Z}$ the map

$$
\gamma_{k}: \mathbb{C}^{*} \rightarrow \widetilde{G}, t \mapsto\left(t^{k}, t^{k-1}\right)
$$

gives a splitting of (2.4) , and each choice of $k$ gives an isomorphism $G \times \mathbb{C}^{*} \cong$ $\widetilde{G}$.

Suppose that the $G$-action on $X$ extends to a $\widetilde{G}$-action on it. By choosing a splitting (2.5), this is equivalent to giving an auxiliary $\mathbb{C}^{*}$-action on $X$ which commutes with the $G$-action. In particular, such a $\widetilde{G}$-action must preserve the $\mathrm{KN}$ stratification (2.1). Let $w \in \Gamma\left(X, \mathcal{O}_{X}\right)$ be $\tau$-semi invariant, i.e. $g^{*} w=\tau(g) w$ for any $g \in \widetilde{G}$. Equivalently $w$ is $G$-invariant, so is a map of stacks

$$
w: \mathcal{X}=[X / G] \rightarrow \mathbb{A}^{1}
$$

and $\mathbb{C}^{*}$-weight one for any choice of splitting (2.5). Given data as above, the derived factorization category

$$
D_{\mathbb{C}^{*}}(\mathcal{X}, w)
$$

is defined to be the triangulated category, whose objects consist of $\widetilde{G}$ equivariant factorizations of $w$, i.e. sequences of $\widetilde{G}$-equivariant morphisms of $\widetilde{G}$-equivariant coherent sheaves $\mathcal{P}_{0}, \mathcal{P}_{1}$ on $X$

$$
\mathcal{P}_{0} \stackrel{f}{\rightarrow} \mathcal{P}_{1} \stackrel{g}{\rightarrow} \mathcal{P}_{0}\langle 1\rangle
$$

satisfying the following:

$$
f \circ g=\cdot w, g \circ f=\cdot w
$$

Here $\langle n\rangle$ means the twist by the $\widetilde{G}$-character $\tau^{n}$. The category (2.6) is defined to be the localization of the homotopy category of the factorizations (2.7) by its subcategory of acyclic factorizations. For details, see [EP15].

2.3. Knörrer periodicity. For the later use, we recall a version of Knörrer periodicity of derived factorization categories proved in [Isi13, Shi12, Hir17] in a general setting. Let $Y$ be a smooth variety with a $G$-action, and $\mathcal{F} \rightarrow$ $Y$ be an algebraic $G$-equivariant vector bundle. Let $\psi: Y \rightarrow \mathcal{F}$ be a $G$ equivariant regular section of it, i.e. its zero locus

$$
Z:=(\psi=0) \subset Y
$$

has codimension equals to the rank of $\mathcal{F}$. The section $\psi$ naturally defines the morphism

$$
w_{\psi}: \mathcal{F}^{\vee} \rightarrow \mathbb{A}^{1}
$$


by sending $(y, v)$ for $y \in Y$ and $\left.v \in \mathcal{F}\right|_{y} ^{\vee}$ to $\langle\psi(y), v\rangle$. We have the following diagram

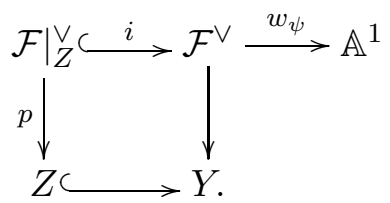

We have the following lemma which is obvious from the definition of $w_{\psi}$ :

Lemma 2.2. For a regular section $\psi$, suppose that $Z$ is non-singular. Then we have $\left\{d w_{\psi}=0\right\}=Z$. Here $Z$ is embedded into $\mathcal{F}^{\vee}$ by the zero section $Y \rightarrow \mathcal{F}^{\vee}$.

The following is the version of Knörrer periodicity we use:

Theorem 2.3. ([Isi13, Shi12, Hir17]) Suppose that the G-action on $\mathcal{F}^{\vee}$ extends to a $\widetilde{G}$-action such that, for some splitting $\gamma_{k}$ in (2.5), the $\mathbb{C}^{*}$ acts on fibers of $\mathcal{F}^{\vee} \rightarrow Y$ with weight one. Then we have the equivalences

$$
D^{b}([Z / G]) \underset{i_{*} \circ p^{*}}{\sim} D^{b}\left(\left[\mathcal{F}^{\vee} /\left(G \times \mathbb{C}^{*}\right)\right], w_{\psi}\right) \underset{\gamma_{k}}{\stackrel{\sim}{\longrightarrow}} D_{\mathbb{C}^{*}}\left(\left[\mathcal{F}^{\vee} / G\right], w_{\psi}\right) .
$$

2.4. Window subcategories. We return to the situation in Section 2.1, 2.2. For a KN-stratification (2.1), let $\eta_{\alpha} \in \mathbb{Z}_{\geq 0}$ be defined by

$$
\eta_{\alpha}=\text { weight }_{\lambda_{\alpha}}\left(\operatorname{det}\left(N_{S_{\alpha} / X}^{\vee}\right)\right) \text {. }
$$

Definition 2.4. For $\delta \in \operatorname{Pic}_{G}(X)_{\mathbb{R}}$, the window subcategory

$$
\mathcal{C}_{\delta} \subset D_{\mathbb{C}^{*}}(\mathcal{X}, w)
$$

is defined to be the triangulated subcategory consisting of factorizations (2.7) such that each derived restriction $\mathcal{P}_{\bullet} \mid z_{\alpha}$ is isomorphic to a factorization $\mathcal{Q}_{\bullet}, \alpha$ of $\left.w\right|_{Z_{\alpha}}$ satisfying the condition

$$
\text { weight }_{\lambda_{\alpha}}\left(\mathcal{Q}_{\bullet, \alpha}\right) \subset \text { weight }_{\lambda_{\alpha}}\left(\left.\delta\right|_{Z_{\alpha}}\right)+\left[-\frac{\eta_{\alpha}}{2}, \frac{\eta_{\alpha}}{2}\right) .
$$

Here we note that weight ${ }_{\lambda_{\alpha}}(\mathcal{Q} \bullet, \alpha)$ is a set of integers.

The following is a version of window theorem for derived categories of GIT quotients.

Theorem 2.5. ([HL15, BFK19]) The composition

$$
\mathcal{C}_{\delta} \subset D_{\mathbb{C}^{*}}(\mathcal{X}, w) \stackrel{\text { res }}{\rightarrow} D_{\mathbb{C}^{*}}\left(\mathcal{X}_{\mathrm{ss}},\left.w\right|_{X_{\mathrm{ss}}}\right)
$$

is an equivalence of triangulated categories. Here the right arrow is the restriction functor to the open substack $\mathcal{X}_{\mathrm{ss}} \subset \mathcal{X}$.

Proof. A version of Theorem 2.5 without an auxiliary $\mathbb{C}^{*}$-action is stated in [BFK19, Corollary 3.2.2, Proposition 3.3.2] together with [BFK19, Remark 3.2.10] (also see [HL15, Proposition 5.5, Example 5.7] for a version of singularity categories). The same arguments apply in the presence of $\mathbb{C}^{*}$-action without any modification. 
2.5. Variation of GIT quotients for linear representations. In the above situation, suppose furthermore that $X$ is a linear representation of $\widetilde{G}$ which decomposes into

$$
X=X_{0} \oplus X_{1}
$$

as $\widetilde{G}$-representations such that $X_{0}$ is a quasi-symmetric $G$-representation. Here a $G$-representation $X_{0}$ is called quasi-symmetric if $\beta_{i} \in M$ for $1 \leq i \leq$ $\operatorname{dim} X_{0}$ are the $T$-weights of $X_{0}^{\vee}$, then for any line $l \subset M_{\mathbb{R}}$ we have

$$
\sum_{\beta_{i} \in l} \beta_{i}=0 .
$$

In particular, a symmetric representation is quasi-symmetric. Let $\bar{\Sigma} \subset M_{\mathbb{R}}$ be the convex hull of the $T$-characters of $\bigwedge^{*}\left(X_{0}^{\vee}\right)$. Then an element $\chi \in M_{\mathbb{R}}$ is called generic if it is contained in the linear span of $\bar{\Sigma}$ but is not parallel to any face of $\bar{\Sigma}$.

Let $\chi_{0}: G \rightarrow \mathbb{C}^{*}$ be a $G$-character. We consider KN stratifications of $X$ and $\mathcal{X}=[X / G]$ with respect to $\mathcal{O}\left(\chi_{0}^{ \pm 1}\right)$

$$
X=X_{\mathrm{ss}}^{ \pm} \sqcup S_{1}^{ \pm} \sqcup S_{2}^{ \pm} \sqcup \cdots, \quad \mathcal{X}=\mathcal{X}_{\mathrm{ss}}^{ \pm} \sqcup \mathcal{S}_{1}^{ \pm} \sqcup \mathcal{S}_{2}^{ \pm} \sqcup \cdots
$$

and take the associated window subcategories for $\delta \in \operatorname{Pic}_{G}(X)_{\mathbb{R}}=M_{\mathbb{R}}^{W}$

$$
\mathcal{C}_{\delta}^{ \pm} \subset D_{\mathbb{C}^{*}}(\mathcal{X}, w) .
$$

We denote by $\left(X_{0}\right)_{\mathrm{ss}}^{ \pm} \subset X_{0}$ the open subsets of $\mathcal{O}\left(\chi_{0}^{ \pm 1}\right)$-semistable points in $X_{0}$. Note that by the definition of GIT stability, we have

$$
\left(X_{0}\right)_{\mathrm{ss}}^{ \pm} \times X_{1} \subset X_{\mathrm{ss}}^{ \pm} \text {. }
$$

We have the following:

Proposition 2.6. Suppose that $\chi_{0} \in M_{\mathbb{R}}^{W}$ is generic and the following condition holds:

$$
X_{\mathrm{ss}}^{-}=\left(X_{0}\right)_{\mathrm{ss}}^{-} \times X_{1} .
$$

Then for any $\delta_{0} \in M_{\mathbb{R}}^{W}$ and $0<\varepsilon \ll 1$, by setting $\delta_{1}=\delta_{0}+\varepsilon \cdot \chi_{0}$ we have $\mathcal{C}_{\delta_{1}}^{-} \subset \mathcal{C}_{\delta_{1}}^{+}$.

Proof. The proposition is proved by applying the argument of Magic window theorem for quasi-symmetric representations [HLS], proved using combinatorial arguments in vVdB17]. Following [HLS, Section 2.2], we define

$$
\mathbb{L}:=\left[X_{0}^{\vee}\right]-\left[\mathfrak{g}^{\vee}\right] \in K_{0}(\operatorname{Rep}(T))=\mathbb{Z}[M] .
$$

For any one parameter subgroup $\lambda: \mathbb{C}^{*} \rightarrow T$, we define $\mathbb{L}^{\lambda>0}$ to be the projection of this class onto the subspace spanned by weights which pair positively with $\lambda$. We define

$$
\bar{\eta}_{\lambda}:=\left\langle\lambda, \mathbb{L}^{\lambda>0}\right\rangle \in \mathbb{Z} .
$$


Then we define $\bar{\nabla} \subset M_{\mathbb{R}}$ by

$$
\bar{\nabla}:=\left\{\chi \in M_{\mathbb{R}}:\langle\lambda, \chi\rangle \in\left[-\frac{\bar{\eta}_{\lambda}}{2}, \frac{\bar{\eta}_{\lambda}}{2}\right] \text { for all } \lambda: \mathbb{C}^{*} \rightarrow T\right\} .
$$

For a region $\Omega \subset M_{\mathbb{R}}$, we denote by

$$
\mathcal{M}(\Omega) \subset D_{\mathbb{C}^{*}}(\mathcal{X}, w)
$$

the triangulated subcategory consisting of factorizations (2.7) such that each $\mathcal{P}_{i}$ is isomorphic to $V \otimes_{\mathbb{C}} \mathcal{O}_{X}$ for a $\widetilde{G}$-representation $V$ whose $T$-weights are contained in $\Omega$. We first claim the inclusion

$$
\mathcal{M}\left(\delta_{1}+\bar{\nabla}\right) \subset \mathcal{C}_{\delta_{1}}^{ \pm}
$$

for $\delta_{1}=\delta_{0}+\varepsilon \cdot \chi_{0}$ with $0<\varepsilon \ll 1$.

Let $\lambda_{\alpha}^{ \pm}: \mathbb{C}^{*} \rightarrow T$ be one parameter subgroups for the KN stratifications (2.9), and $Z_{\alpha}^{ \pm} \subset S_{\alpha}^{ \pm}$the centers. For a $\widetilde{G}$-representation $V$ whose $T$-weights are contained in $\delta_{1}+\bar{\nabla}$, we have

$$
\text { weight }_{\lambda_{\alpha}^{ \pm}}\left(\left.V \otimes_{\mathbb{C}} \mathcal{O}_{X}\right|_{Z_{\alpha}^{ \pm}}\right) \subset\left\langle\delta_{1}, \lambda_{\alpha}^{ \pm}\right\rangle+\left[-\frac{\bar{\eta}_{\lambda_{\alpha}^{ \pm}}}{2}, \frac{\bar{\eta}_{\lambda_{\alpha}^{ \pm}}}{2}\right]
$$

by the definition of $\bar{\nabla}$. We have the inequality

$$
\bar{\eta}_{\lambda_{\alpha}^{ \pm}} \leq\left\langle\lambda_{\alpha}^{ \pm},\left(X^{\vee}\right)^{\lambda_{\alpha}^{ \pm}>0}-\left(\mathfrak{g}^{\vee}\right)^{\lambda_{\alpha}^{ \pm}>0}\right\rangle=\eta_{\alpha}^{ \pm}
$$

where the latter is defined as in (2.8) for the KN stratifications (2.9). For the second equality, see [HL15, Equation (4)]. On the other hand, we have $\left\langle\chi_{0}^{ \pm 1}, \lambda_{\alpha}^{ \pm}\right\rangle<0$ by the property of KN stratifications. Therefore by taking $0<\varepsilon \ll 1$ so that $\left\langle\delta_{1}, \lambda_{\alpha}^{ \pm}\right\rangle+\eta_{\alpha}^{ \pm} / 2$ are not integers, we have

$$
\text { weight }_{\lambda_{\alpha}^{ \pm}}\left(\left.V \otimes_{\mathbb{C}} \mathcal{O}_{X}\right|_{Z_{\alpha}^{ \pm}}\right) \subset\left\langle\delta_{1}, \lambda_{\alpha}^{ \pm}\right\rangle+\left[-\frac{\eta_{\alpha}^{ \pm}}{2}, \frac{\eta_{\alpha}^{ \pm}}{2}\right) .
$$

Therefore the inclusion (2.13) holds.

We consider the following commutative diagram

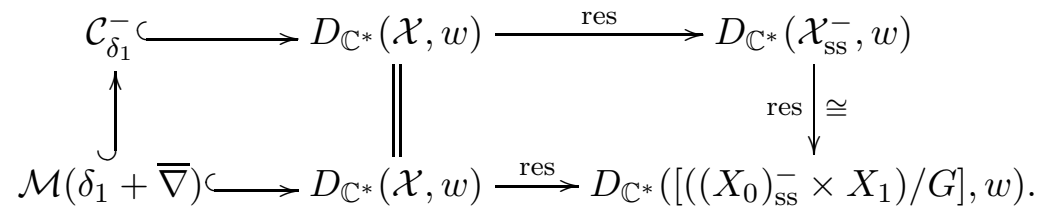

The top composition is an equivalence by Theorem 2.5 and the right restriction functor is an equivalence by the assumption (2.10).

Below we show that the bottom composition of the diagram (2.14) is essentially surjective. Let

$$
\mathcal{M}^{\prime}\left(\delta_{1}+\bar{\nabla}\right) \subset D^{b}\left(\left[X_{0} / \widetilde{G}\right]\right), \mathcal{M}^{\prime \prime}\left(\delta_{1}+\bar{\nabla}\right) \subset D^{b}([X / \widetilde{G}])
$$

be the triagulated subcategories generated by $\widetilde{G}$-equivariant vector bundles on $X_{0}, X$ whose $T$-weights are contained in $\delta_{1}+\bar{\nabla}$. By [HLS, Corollary 2.9], any facet of $\bar{\nabla}$ is parallel to some facet of $\bar{\Sigma}$. Therefore the genericity 
condition of $\chi_{0}$ implies that $\partial\left(\delta_{1}+\bar{\nabla}\right) \cap M=\emptyset$, and $\left[\left(X_{0}\right)_{T-\text { ss }}^{-} / T\right]$ is DeligneMumford by [HLS, Proposition 2.1]. Therefore the assumption of [HLS, Proposition 3.11] is satisfied, which shows that the following composition is an equivalence (Magic window theorem in [HLS, Theorem 3.2])

$$
\left.\mathcal{M}^{\prime}\left(\delta_{1}+\bar{\nabla}\right) \hookrightarrow D^{b}\left(\left[X_{0} / \widetilde{G}\right]\right) \rightarrow D^{b}\left(\left[\left(X_{0}\right)_{\mathrm{ss}}^{-} / \widetilde{G}\right)\right]\right) .
$$

The above result is stated in loc. cit. without an auxiliary $\mathbb{C}^{*}$-action, but the same argument applies in the presence of an auxiliary $\mathbb{C}^{*}$-action, see [HLS, Corollary 5.2].

Also $D^{b}\left(\left[\left(\left(X_{0}\right)_{\mathrm{ss}}^{-} \times X_{1}\right) / \widetilde{G}\right]\right)$ is generated by images of the pull-backs by the projection

$$
\left[\left(\left(X_{0}\right)_{\mathrm{ss}}^{-} \times X_{1}\right) / \widetilde{G}\right] \rightarrow\left[\left(X_{0}\right)_{\mathrm{ss}}^{-} / \widetilde{G}\right]
$$

as it is a total space of a vector bundle on $\left[\left(X_{0}\right)_{\mathrm{sS}}^{-} / \widetilde{G}\right]$. It follows that $D^{b}\left(\left[\left(\left(X_{0}\right)_{\mathrm{ss}}^{-} \times X_{1}\right) / \widetilde{G}\right]\right)$ is generated by $V \otimes_{\mathbb{C}} \mathcal{O}_{X}$ for $\widetilde{G}$-representation $V$ whose $T$-weights are contained in $\delta_{1}+\bar{\nabla}$. In other words, the composition

$$
\mathcal{M}^{\prime \prime}\left(\delta_{1}+\bar{\nabla}\right) \hookrightarrow D^{b}([X / \widetilde{G}]) \rightarrow D^{b}\left(\left[\left(X_{0}\right)_{\mathrm{ss}}^{-} \times X_{1} / \widetilde{G}\right]\right)
$$

is essentially surjective. The above composition is also fully-faithful by a version of the diagram (2.14) without $w$, so the functor (2.15) is an equivalence.

By the equivalence (2.15), we see that any $\widetilde{G}$-equivariant factorization on $\left(X_{0}\right)_{\mathrm{ss}}^{-} \times X_{1}$ is a restriction of some object in $\mathcal{M}\left(\delta_{1}+\bar{\nabla}\right)$. Indeed for a factorization

$$
\mathcal{P}_{0} \stackrel{f}{\rightarrow} \mathcal{P}_{1} \stackrel{g}{\rightarrow} \mathcal{P}_{0}\langle 1\rangle
$$

on $\left(X_{0}\right)_{\mathrm{sS}}^{-} \times X_{1}$, each $\mathcal{P}_{i}$ is resolved by a complex of vector bundles $\mathcal{V}_{i}^{\bullet}$ in $\mathcal{M}^{\prime \prime}\left(\delta_{1}+\bar{\nabla}\right)$ restricted to $\left(X_{0}\right)_{\mathrm{ss}}^{-} \times X_{1}$. By the equivalence (2.15), the morphisms $f, g$ can be lifted to morphisms of complexes $\mathcal{V}_{0}^{\bullet} \rightarrow \mathcal{V}_{1}^{\bullet} \rightarrow \mathcal{V}_{0}^{\bullet}\langle 1\rangle$ on $X$. By taking the totalizations, we obtain a factorization in $\mathcal{M}\left(\delta_{1}+\bar{\nabla}\right)$ whose restriction to $\left(X_{0}\right)_{\mathrm{ss}}^{-} \times X_{1}$ gives (2.16) . Therefore the bottom arrow of (2.14) is essentially surjective. The above arguments show that $\mathcal{C}_{\delta_{1}}^{-}=$ $\mathcal{M}\left(\delta_{1}+\bar{\nabla}\right) \subset \mathcal{C}_{\delta_{1}}^{+}$as desired.

We will show that a similar result of Proposition 2.6 also holds after taking the direct sum with some symmetric representations. Let $W$ be a finite dimensional $G$-representation whose weights of $\mathbb{C}^{*} \subset G$ are one. We set

$$
X_{W}=X \oplus W \oplus W^{\vee}, \mathcal{X}_{W}=\left[X_{W} / G\right] .
$$

We have the KN stratification for the $G$-action on $X_{W}$ with respect to $\mathcal{O}\left(\chi_{0}^{ \pm 1}\right)$

$$
X_{W}=\left(X_{W}\right)_{\mathrm{ss}}^{ \pm} \sqcup S_{W, 1}^{ \pm} \sqcup S_{W, 2}^{ \pm} \cdots, \mathcal{X}_{W}=\left(\mathcal{X}_{W}\right)_{\mathrm{ss}}^{ \pm} \sqcup \mathcal{S}_{W, 1}^{ \pm} \sqcup \mathcal{S}_{W, 2}^{ \pm} \cdots
$$


The $G$-action on $X$ is naturally extended to a $\widetilde{G}$-action on $X_{W}$ given by

$$
\left(g, g^{\prime}\right)\left(x, v, v^{\prime}\right)=\left(\left(g, g^{\prime}\right)(x), g(v), g^{\prime}\left(v^{\prime}\right)\right)
$$

for $x \in X, v \in W$ and $v^{\prime} \in W^{\vee}$. Let $w^{\star}$ be given by

$$
w^{\star}: X_{W} \rightarrow \mathbb{C}, w^{\star}\left(x, v, v^{\prime}\right)=w(x)+\left\langle v, v^{\prime}\right\rangle .
$$

For $\delta \in M_{\mathbb{R}}^{W}$, we have the associated window subcategories with respect to the stratifications (2.17)

$$
\mathcal{C}_{W, \delta}^{ \pm} \subset D_{\mathbb{C}^{*}}\left(\mathcal{X}_{W}, w^{\star}\right)
$$

Theorem 2.7. Under the same assumption of Proposition 2.6, we have $\mathcal{C}_{W, \delta_{2}}^{-} \subset \mathcal{C}_{W, \delta_{2}}^{+}$for $\delta_{2}=\varepsilon \cdot \chi_{0}$ with $0<\varepsilon \ll 1$.

Proof. Note that if $\left(X_{W}\right)_{\mathrm{ss}}^{-}=\left(X_{0} \times W \times W^{\vee}\right)_{\mathrm{ss}}^{-} \times X_{1}$, then the theorem follows from Proposition 2.6. However this is not the case in general, and we will prove the theorem by comparing magic windows under the Knörrer periodicity equivalence. Below, we use the notation in the proof of Proposition 2.6.

Let $\mathbb{L}_{W}$ be defined by

$$
\mathbb{L}_{W}=\left[X_{0}^{\vee}\right]+[W]+\left[W^{\vee}\right]-\left[\mathfrak{g}^{\vee}\right] \in K(\operatorname{Rep}(T)) .
$$

Then for each one parameter subgroup $\lambda: \mathbb{C}^{*} \rightarrow T$, we set

$$
\begin{aligned}
\bar{\eta}_{W, \lambda} & :=\left\langle\lambda, \mathbb{L}_{W}^{\lambda>0}\right\rangle \\
& =\bar{\eta}_{\lambda}+\left\langle\lambda, W^{\lambda>0}\right\rangle-\left\langle\lambda, W^{\lambda<0}\right\rangle .
\end{aligned}
$$

We set $\bar{\nabla}_{W}$ to be

$$
\bar{\nabla}_{W}:=\left\{\chi \in M_{\mathbb{R}}:\langle\lambda, \chi\rangle \in\left[-\frac{\bar{\eta}_{W, \lambda}}{2}, \frac{\bar{\eta}_{W, \lambda}}{2}\right] \text { for all } \lambda: \mathbb{C}^{*} \rightarrow T\right\} .
$$

For $\delta \in M_{\mathbb{R}}^{W}$, we have the subcategory

$$
\mathcal{M}\left(\delta+\bar{\nabla}_{W}\right) \subset D_{\mathbb{C}^{*}}\left(\mathcal{X}_{W}, w^{\star}\right)
$$

similarly to (2.12). By the argument of Proposition 2.6, we always have the inclusion $\mathcal{M}\left(\delta+\bar{\nabla}_{W}\right) \subset \mathcal{C}_{W, \delta}^{ \pm}$. It is enough to show that $\mathcal{M}\left(\delta_{2}+\bar{\nabla}_{W}\right)=\mathcal{C}_{W, \delta_{2}}^{-}$ for $\delta_{2}=\epsilon \cdot \chi_{0}$ with $0<\varepsilon \ll 1$.

We have the following commutative diagram

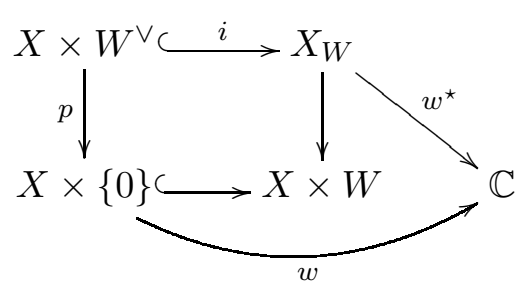


Here each vertical arrow is a projection. The above diagram induces the Knörrer periodicity equivalence (see [Hir17, Theorem 4.2])

$$
\Phi:=i_{*} \circ p^{*}: D_{\mathbb{C}^{*}}(\mathcal{X}, w) \stackrel{\sim}{\rightarrow} D_{\mathbb{C}^{*}}\left(\mathcal{X}_{W}, w^{\star}\right) .
$$

The above equivalence is nothing but taking the tensor product over $\mathcal{O}_{X}$ with the factorization

$$
\mathcal{O}_{X \times W} \vee \rightarrow 0 \rightarrow \mathcal{O}_{X \times W} \vee\langle 1\rangle
$$

of the function $w^{\prime}:\left(x, v, v^{\prime}\right) \mapsto\left\langle v, v^{\prime}\right\rangle$ on $X_{W}$. The above factorization is isomorphic to the Koszul factorization on $X_{W}$ of $w^{\prime}$ of the form (see BFK14, Proposition 3.20])

$$
\bigwedge_{\text {even }} W \otimes \mathcal{O}_{X_{W}} \rightarrow \bigwedge^{\text {odd }} W \otimes \mathcal{O}_{X_{W}} \rightarrow \bigwedge^{\text {even }} W \otimes \mathcal{O}_{X_{W}}\langle 1\rangle
$$

The Koszul factorization (2.21) has the minimus $T$-weight $\left\langle\lambda, W^{\lambda<0}\right\rangle$ and the maximum $T$-weight $\left\langle\lambda, W^{\lambda}>0\right\rangle$. Therefore for each $\mathcal{E} \in \mathcal{M}\left(\delta_{1}+\bar{\nabla}\right)$ in $D_{\mathbb{C}^{*}}(\mathcal{X}, w)$, where $\delta_{1}=\delta_{0}+\varepsilon \cdot \chi_{0}$, a $T$-weight $\chi$ of $\Phi(\mathcal{E})$ satisfies that

$$
-\frac{1}{2} \bar{\eta}_{\lambda}+\left\langle\lambda, W^{\lambda<0}\right\rangle \leq\left\langle\chi-\delta_{1}, \lambda\right\rangle \leq \frac{1}{2} \bar{\eta}_{\lambda}+\left\langle\lambda, W^{\lambda>0}\right\rangle .
$$

By the identity (2.18), we have

$$
\begin{aligned}
\frac{1}{2} \bar{\eta}_{\lambda}+\left\langle\lambda, W^{\lambda>0}\right\rangle & =\frac{1}{2} \bar{\eta}_{W, \lambda}+\frac{1}{2}\left\langle\lambda, W^{\lambda>0}\right\rangle+\frac{1}{2}\left\langle\lambda, W^{\lambda<0}\right\rangle \\
& =\frac{1}{2} \bar{\eta}_{W, \lambda}+\frac{1}{2} \text { weight }_{\lambda}(\operatorname{det} W) .
\end{aligned}
$$

Similarly we have

$$
-\frac{1}{2} \bar{\eta}_{\lambda}+\left\langle\lambda, W^{\lambda<0}\right\rangle=-\frac{1}{2} \bar{\eta}_{W, \lambda}+\frac{1}{2} \text { weight }_{\lambda}(\operatorname{det} W) .
$$

We take $\delta_{0}=-\frac{1}{2} \operatorname{det}(W) \in \operatorname{Pic}(\mathcal{X})_{\mathbb{R}}=M_{\mathbb{R}}^{W}$. Then the above argument implies that

$$
-\frac{1}{2} \bar{\eta}_{W, \lambda} \leq\left\langle\chi-\delta_{2}, \lambda\right\rangle \leq \frac{1}{2} \bar{\eta}_{W, \lambda}
$$

This means that the functor $\Phi$ restricts to the functor

$$
\Phi: \mathcal{M}\left(\delta_{1}+\bar{\nabla}\right) \rightarrow \mathcal{M}\left(\delta_{2}+\bar{\nabla}_{W}\right)
$$


We have the commutative diagram

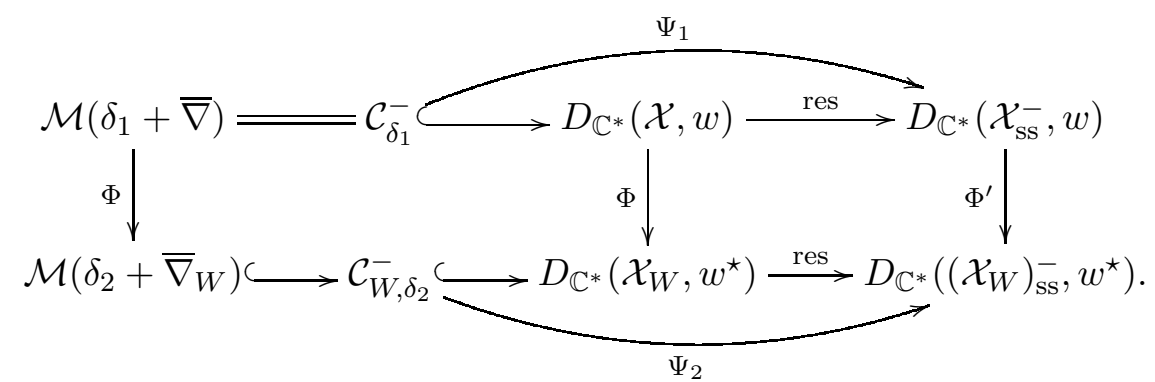

Here the top left identity is proved in the proof of Proposition 2.6, and $\Psi_{1}$, $\Psi_{2}$ are equivalences by Theorem 2.5 .

We see that the equivalence $\Phi$ descends to the equivalence $\Phi^{\prime}$ in the diagram (2.22). Since we have

$$
\left\{d w^{\star}=0\right\}=\{d w=0\} \times\{0\} \times\{0\} \subset X \times W \times W^{\vee}
$$

we have

$$
\left\{d w^{\star}=0\right\} \cap\left(X_{W}\right)_{\mathrm{ss}}^{-} \subset X_{\mathrm{ss}}^{-} \times W \times W^{\vee} .
$$

The equivalence $\Phi^{\prime}$ is given by the composition of equivalences

$$
\Phi^{\prime}: D_{\mathbb{C}^{*}}\left(\mathcal{X}_{\mathrm{ss}}^{-}, w\right) \stackrel{\sim}{\rightarrow} D_{\mathbb{C}^{*}}\left(\left[X_{\mathrm{Ss}}^{-} \times W \times W^{\vee} / G\right], w^{\star}\right) \stackrel{\sim}{\rightarrow} D_{\mathbb{C}^{*}}\left(\left(\mathcal{X}_{W}\right)_{\mathrm{ss}}^{-}, w^{\star}\right) .
$$

Here the first equivalence is the Knörrer periodicity similar to (2.20), and the second equivalence follows from (2.23) together with the fact that the derived factorization category only depends on an open neighborhood of the critical locus (for example see [HLS, Lemma 5.5]). The resulting equivalence $\Phi^{\prime}$ fits into the commutative diagram (2.22) by the construction.

By the diagram (2.22), we have the inclusion $\Phi\left(\mathcal{C}_{\delta_{1}}^{-}\right) \subset \mathcal{C}_{W, \delta_{2}}^{-}$. Since $\Psi_{1}$, $\Psi_{2}$ and $\Phi^{\prime}$ are equivalences, it follows that $\Phi\left(\mathcal{C}_{\delta_{1}}^{-}\right)=\mathcal{C}_{W, \delta_{2}}^{-}$. Again using the diagram (2.22), we conclude that $\mathcal{C}_{W, \delta_{2}}^{-}=\mathcal{M}\left(\delta_{2}+\bar{\nabla}_{W}\right)$ as desired.

We have the obvious corollary of Theorem 2.7 ,

Corollary 2.8. Suppose that $\chi_{0} \in M_{\mathbb{R}}^{W}$ is generic and the following condition holds:

$$
X_{\mathrm{ss}}^{ \pm}=\left(X_{0}\right)_{\mathrm{ss}}^{ \pm} \times X_{1}
$$

Then for any finite dimensional $G$-representation $W$, we have $\mathcal{C}_{W, \delta_{2}}^{-}=\mathcal{C}_{W, \delta_{2}}^{+}$ for $\delta_{2}=\varepsilon \cdot \chi_{0}$ with $0<\varepsilon \ll 1$.

\section{DERIVED CATEGORIES OF REPRESENTATIONS OF QUIVERS}

In this section, we study derived factorization categories associated with certain quivers with super-potentials, and show an inclusion of the window subcategories under wall-crossing. The result of this section is a local version of Theorem 1.1. 
3.1. Representations of quivers. Let $Q$ be a quiver

$$
Q=(V(Q), E(Q), s, t)
$$

where $V(Q)$ is the set of vertices, $E(Q)$ is the set of edges, and $s, t: E(Q) \rightarrow$ $V(Q)$ are maps which correspond to sources and targets of edges. For $e \in$ $E(Q)$ with $s(e)=i, t(e)=j$, we write $e=(i \rightarrow j)$. Below we assume that $Q$ is symmetric, i.e.

$$
\sharp(i \rightarrow j)=\sharp(j \rightarrow i), i, j \in V(Q) .
$$

We fix finite dimensional vector spaces $V_{i}$ for each $i \in V(Q)$, and set

$$
\operatorname{Rep}(Q)=\prod_{(i \rightarrow j) \in E(Q)} \operatorname{Hom}\left(V_{i}, V_{j}\right),
$$

The algebraic group $G$ defined by

$$
G=\prod_{i \in V(Q)} \mathrm{GL}\left(V_{i}\right)
$$

acts on $\operatorname{Rep}(Q)$ by the conjugation, which descends to the action of $\mathbb{P}(G)=$ $G / \mathbb{C}^{*}$ where $\mathbb{C}^{*} \subset G$ is the diagonal torus. We have the quotient stack together with its good moduli space

$$
p_{Q}: \operatorname{Rep}(Q) \rightarrow[\operatorname{Rep}(Q) / G] \stackrel{\iota Q}{\rightarrow} \operatorname{Rep}(Q) / / G .
$$

The stack $[\operatorname{Rep}(Q) / G]$ is the moduli stack of $Q$-representations with dimension vector $\left(\operatorname{dim} V_{i}\right)_{i \in Q}$, and $\operatorname{Rep}(Q) / / G$ parametrizes semisimple $Q$ representations with the above dimension vector.

3.2. Representations of extended quivers. For each $i \in V(Q)$, let us take finite sets

$$
\mathbf{E}_{i 0} \subset \mathbf{E}_{0 i} .
$$

We define the extended quiver $Q^{\dagger}$ by setting

$$
V\left(Q^{\dagger}\right)=V(Q) \sqcup\{0\}, E\left(Q^{\dagger}\right)=E(Q) \sqcup_{i \in V(Q)} \mathbf{E}_{0 i} \sqcup_{i \in V(Q)} \mathbf{E}_{i 0} .
$$

Here for $e \in \mathbf{E}_{0 i}$ (resp. $e \in \mathbf{E}_{i 0}$ ), its source and target are 0, $i$ (resp. $i, 0$ ) respectively. We set

$$
\operatorname{Rep}\left(Q^{\dagger}\right)=\operatorname{Rep}(Q) \times \prod_{(0 \rightarrow i) \in \mathbf{E}_{0 i}} V_{i} \times \prod_{(i \rightarrow 0) \in \mathbf{E}_{i 0}} V_{i}^{\vee} .
$$

The space $\operatorname{Rep}\left(Q^{\dagger}\right)$ parametrizes $Q^{\dagger}$-representations with dimension vector $\left(\left(\operatorname{dim} V_{i}\right)_{i \in V(Q)}, 1\right)$.

As defined in (2.3), let $\widetilde{G}=G \times_{\mathbb{P}(G)} G$. There is a natural $\widetilde{G}$-action on $\operatorname{Rep}\left(Q^{\dagger}\right)$ given by

$$
\left(g, g^{\prime}\right) \cdot\left(x, u, u^{\prime}\right)=\left(g(x), g(u), g^{\prime}\left(u^{\prime}\right)\right)=\left(g^{\prime}(x), g(u), g^{\prime}\left(u^{\prime}\right)\right) .
$$

Here $\left(g, g^{\prime}\right) \in \widetilde{G}$ and the second identity follows as the $G$-action on $\operatorname{Rep}(Q)$ descends to the $\mathbb{P}(G)$-action. 
Let $\chi_{0}: G \rightarrow \mathbb{C}^{*}$ be the character of $G$ defined by

$$
\chi_{0}\left(\left(g_{i}\right)_{i \in V(Q)}\right) \mapsto \prod_{i \in Q_{0}} \operatorname{det}\left(g_{i}\right) .
$$

We have the open substacks of semistable locus with respect to $\mathcal{O}\left(\chi_{0}^{ \pm 1}\right)$

$$
M_{Q^{\dagger}}^{ \pm}:=\left[\operatorname{Rep}\left(Q^{\dagger}\right)_{\mathrm{ss}}^{ \pm} / G\right] \subset\left[\operatorname{Rep}\left(Q^{\dagger}\right) / G\right] .
$$

Proposition 3.1. The stacks $M_{Q^{\dagger}}^{ \pm}$are smooth varieties and, if $M_{Q^{\dagger}}^{-} \neq \emptyset$, then the diagram

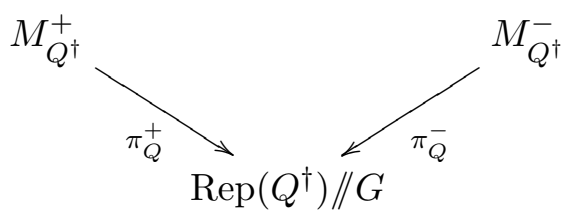

is a flip if $\sharp \mathbf{E}_{i 0}<\sharp \mathbf{E}_{0 i}$ for all $i \in V(Q)$. It is a flop if $\sharp \mathbf{E}_{i 0}=\sharp \mathbf{E}_{0 i}$ for all $i \in V(Q)$.

Proof. By a correspondence of GIT stability and King's $\theta$-stability [Kin94, we see that a $Q^{\dagger}$-representation in $\operatorname{Rep}\left(Q^{\dagger}\right)$ is $\mathcal{O}\left(\chi_{0}^{ \pm 1}\right)$-semistable if and only if it is $\theta^{ \pm}$-semistable where

$$
\theta^{ \pm}=\left(\theta_{i}^{ \pm}\right)_{i \in V\left(Q^{\dagger}\right)}, \theta_{i}^{ \pm}=\mp 1 \text { for } i \in V(Q), \theta_{0}^{ \pm}= \pm \sum_{i \in V(Q)} \operatorname{dim} V_{i} .
$$

Here a $Q^{\dagger}$-representation $E$ is $\theta^{ \pm}$-semistable if for any subrepresentation $F \subset E$, we have $\theta^{ \pm}(\underline{\operatorname{dim}} F) \geq \theta^{ \pm}(\underline{\operatorname{dim}} E)=0$, where $\underline{\operatorname{dim}}$ is the dimension vector. Then the proposition follows from [Toda, Proposition 7.13].

3.3. Super-potentials on quiver representations. Suppose that we are given a $G$-equivariant morphism of $G$-equivariant vector bundles on $\operatorname{Rep}(Q)$

$$
\vartheta:\left(\prod_{(0 \rightarrow i) \in \mathbf{E}_{0 i}} V_{i}\right) \otimes \mathcal{O}_{\operatorname{Rep}(Q)} \rightarrow\left(\prod_{(i \rightarrow 0) \in \mathbf{E}_{i 0}} V_{i}\right) \otimes \mathcal{O}_{\operatorname{Rep}(Q)} .
$$

Then the above morphism induces the function

$$
w: \operatorname{Rep}\left(Q^{\dagger}\right) \rightarrow \mathbb{C}, w\left(x, u, u^{\prime}\right)=\left\langle\left.\vartheta\right|_{x}(u), u^{\prime}\right\rangle .
$$

for $x \in \operatorname{Rep}(Q), u \in \prod_{(0 \rightarrow i) \in \mathbf{E}_{0 i}} V_{i}, u^{\prime} \in \prod_{(i \rightarrow 0) \in \mathbf{E}_{i 0}} V_{i}^{\vee}$. The function $w$ is $G$-invariant, so descends to the map

$$
w^{0}: \operatorname{Rep}\left(Q^{\dagger}\right) / / G \rightarrow \mathbb{C} .
$$

We define $w^{ \pm}$by the commutative diagram

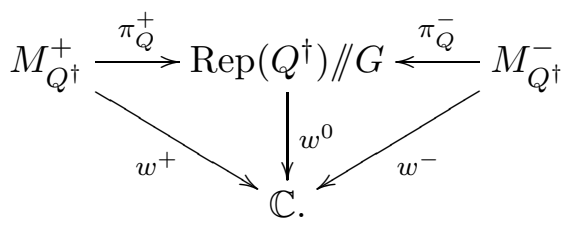


We define the following d-critical loci

$$
M_{\left(Q^{\dagger}, w\right)}^{ \pm}:=\left\{d w^{ \pm}=0\right\} \subset M_{Q^{\dagger}}^{ \pm} .
$$

Proposition 3.2. Suppose that the followings hold:

$$
\begin{gathered}
M_{\left(Q^{\dagger}, w\right)}^{+} \subset\left[\left(\operatorname{Rep}(Q) \times \prod_{(0 \rightarrow i) \in \mathbf{E}_{0 i}} V_{i} \times\{0\}\right) / G\right], \\
M_{\left(Q^{\dagger}, w\right)}^{-} \subset\left[\left(\operatorname{Rep}(Q) \times\{0\} \times \prod_{(i \rightarrow 0) \in \mathbf{E}_{i 0}} V_{i}^{\vee}\right) / G\right] .
\end{gathered}
$$

Then we have the diagram

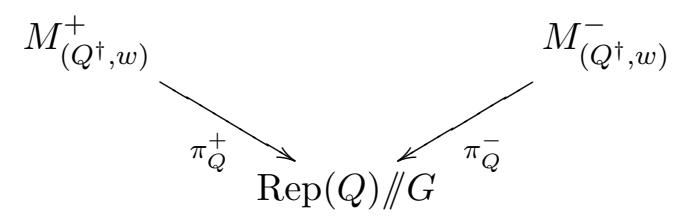

which is a d-critical flip if $\sharp \mathbf{E}_{i 0}<\sharp \mathbf{E}_{0 i}$ for all $i \in V(Q)$. It is a d-critical flop if $\sharp \mathbf{E}_{i 0}=\sharp \mathbf{E}_{0 i}$ for all $i \in V(Q)$.

Proof. The assumption (3.8) implies that $\pi_{Q}^{ \pm}$restricted to $M_{(Q, w)}^{ \pm}$factors through the closed immersion

$$
\operatorname{Rep}(Q) / / G \hookrightarrow \operatorname{Rep}\left(Q^{\dagger}\right) / / G
$$

induced by the zero section of the projection $\operatorname{Rep}\left(Q^{\dagger}\right) \rightarrow \operatorname{Rep}(Q)$. Therefore we have the following diagram

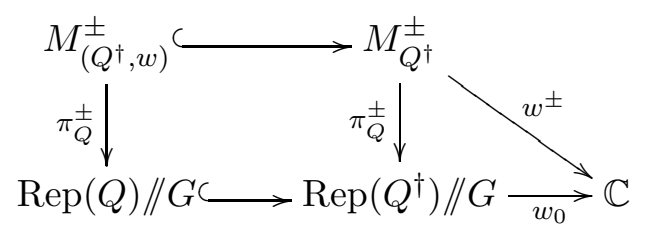

giving relative d-critical charts of $M_{\left(Q^{\dagger}, w\right)}^{ \pm}$(see Definition A.3). Then the proposition follows from Proposition [3.1]

3.4. Relations with symmetric quiver representations. We define the subquiver

$$
Q_{0}^{\dagger} \subset Q^{\dagger}
$$

defined by $V\left(Q_{0}^{\dagger}\right)=V\left(Q^{\dagger}\right)$, the set of edges from $i \in V\left(Q_{0}^{\dagger}\right)$ to $j \in V\left(Q_{0}^{\dagger}\right)$ in $Q_{0}^{\dagger}$ is the same as that in $Q^{\dagger}$ except the case $i=0$ and $j \in V(Q)$ :

$$
\left\{0 \rightarrow i \text { in } Q_{0}^{\dagger}\right\}:=\mathbf{E}_{i 0} \subset \mathbf{E}_{0 i}=\left\{0 \rightarrow i \text { in } Q^{\dagger}\right\} .
$$

The embedding (3.2) realizes $Q_{0}^{\dagger}$ as a subquiver of $Q^{\dagger}$. From its construction, the quiver $Q_{0}^{\dagger}$ is symmetric. Let $\operatorname{Rep}\left(Q_{0}^{\dagger}\right)$ be its representation space with 
dimension vector $\left(\left(\operatorname{dim} V_{i}\right)_{i \in V(Q)}, 1\right)$. We have the following decomposition as $\widetilde{G}$-representation

$$
\operatorname{Rep}\left(Q^{\dagger}\right)=\operatorname{Rep}\left(Q_{0}^{\dagger}\right) \times \prod_{(0 \rightarrow i) \in \mathbf{E}_{0 i} \backslash \mathbf{E}_{i 0}} V_{i} .
$$

Note that $\operatorname{Rep}\left(Q_{0}^{\dagger}\right)$ is a symmetric $G$-representation.

Lemma 3.3. The element $\chi_{0} \in M_{\mathbb{R}}^{W}$ defined by (3.3) is generic with respect to the symmetric $G$-representation $\operatorname{Rep}\left(Q_{0}^{\dagger}\right)$.

Proof. Let $\beta_{1}, \ldots, \beta_{d} \in M$ for $d=\operatorname{dim} \operatorname{Rep}\left(Q_{0}^{\dagger}\right)$ be the $T$-weights of $\operatorname{Rep}\left(Q_{0}^{\dagger}\right)^{\vee}$. By [HLS, Proposition 2.1], the genericity of $\chi_{0}$ is equivalent to that for any proper subspace $H \subsetneq M_{\mathbb{R}}$, there is a one parameter subgroup $\lambda: \mathbb{C}^{*} \rightarrow$ $T$ such that $\left\langle\lambda, \beta_{j}\right\rangle=0$ for any $\beta_{j} \in H$ and $\left\langle\lambda, \chi_{0}\right\rangle \neq 0$. Note that $T=\prod_{i \in V(Q)} T_{i}$ for the maximal torus $T_{i} \subset \mathrm{GL}\left(V_{i}\right)$. By choosing basis $\left\{e_{i 1}, \ldots, e_{i d_{i}}\right\}$ of $V_{i}$, where $d_{i}=\operatorname{dim} V_{i}$, we can write $M_{\mathbb{R}}$ as

$$
M_{\mathbb{R}}=\bigoplus_{i \in V(Q)} \bigoplus_{1 \leq k \leq d_{i}} \mathbb{R} e_{i k}
$$

Then any non-zero $T$-character $\beta_{j}$ of $\operatorname{Rep}\left(Q_{0}^{\dagger}\right)^{\vee}$ is either of the form $\pm e_{i k}$ or $e_{i k}-e_{i^{\prime} k^{\prime}}$. For a proper linear subspace $H \subsetneq M_{\mathbb{R}}$, let $\lambda \in N$ be the cocharacter defined by

$$
\lambda=\left(\lambda_{i k}\right)_{i \in V(Q), 1 \leq k \leq d_{i}}, \quad \lambda_{i k}=\left\{\begin{array}{cc}
0 & e_{i k} \in H \\
1 & e_{i k} \notin H
\end{array}\right.
$$

Then $\left\langle\lambda, \beta_{j}\right\rangle=0$ for any $\beta_{j} \in H$. As $\chi_{0}=\sum_{i, k} e_{i k}$, we have

$$
\left\langle\lambda, \chi_{0}\right\rangle=\sharp\left\{(i, k): i \in V(Q), 1 \leq k \leq d_{i} e_{i k} \notin H\right\}>0
$$

where the latter inequality holds as $H$ is a proper subspace. Therefore $\chi_{0}$ is generic.

We denote by

$$
\operatorname{Rep}\left(Q_{0}^{\dagger}\right)_{\mathrm{ss}}^{ \pm} \subset \operatorname{Rep}\left(Q_{0}^{\dagger}\right)
$$

the semistable locus with respect to the $G$-action and $G$-linearizations $\mathcal{O}\left(\chi_{0}^{ \pm 1}\right)$.

Lemma 3.4. We have the identity

$$
\operatorname{Rep}\left(Q^{\dagger}\right)_{\mathrm{ss}}^{-}=\operatorname{Rep}\left(Q_{0}^{\dagger}\right)_{\mathrm{ss}}^{-} \times \prod_{(0 \rightarrow i) \in \mathbf{E}_{0 i} \backslash \mathbf{E}_{i 0}} V_{i} .
$$

Proof. Let a $Q^{\dagger}$-representation $E$ be represented by a point

$$
x=\left(x_{e}\right)_{e \in E\left(Q^{\dagger}\right)} \in \operatorname{Rep}\left(Q^{\dagger}\right), x_{e}: V_{s(e)} \rightarrow V_{t(e)}
$$

where $V_{0}=\mathbb{C}$. We set

$$
T=\bigoplus_{i \in V(Q)} V_{i}^{\vee} .
$$


Then $T$ has a $\mathbb{C}[Q]$-module structure given by $x_{e}^{\vee}$ for $e \in E(Q)$, which makes sense as $Q$ is symmetric. By the proof of Proposition 3.1, $E$ is semistable with respect to $\mathcal{O}\left(\chi_{0}^{-1}\right)$ if and only if it is $\theta^{-}$-semistable, where $\theta^{-}$is given in (3.4). Then by [Toda, Lemma 7.10], we see that $E$ is $\theta^{-}$-semistable if and only if $T$ is generated as a $\mathbb{C}[Q]$-module by the images of the maps

$$
x_{e}^{\vee}: \mathbb{C} \rightarrow V_{i}^{\vee}, e \in \mathbf{E}_{i 0} .
$$

In particular, the $\theta^{-}$-stability does not impose any constraint on the maps $x_{e}: \mathbb{C} \rightarrow V_{i}$ for $e \in \mathbf{E}_{0 i}$. The same statement applies to $\theta^{-}$-semistable $Q_{0}^{\dagger}$-representations. Therefore (3.9) holds.

3.5. Window subcategories for quiver representations. Let $W$ be a finite dimensional $G$-representation. We set

$$
\operatorname{Rep}\left(Q^{\dagger}\right)_{W}:=\operatorname{Rep}\left(Q^{\dagger}\right) \times W \times W^{\vee} .
$$

The $\widetilde{G}$-action on $\operatorname{Rep}\left(Q^{\dagger}\right)$ extends to the action on $\operatorname{Rep}\left(Q^{\dagger}\right)_{W}$ by

$$
\left(g, g^{\prime}\right) \cdot\left(x, u, u^{\prime}, v, v^{\prime}\right)=\left(g(x), g(u), g^{\prime}\left(u^{\prime}\right), g(v), g^{\prime}\left(v^{\prime}\right)\right) \text {. }
$$

Here $\left(g, g^{\prime}\right) \in \widetilde{G}$ and

$$
\left(x, u, u^{\prime}, v, v^{\prime}\right) \in \operatorname{Rep}(Q) \times\left(\prod_{(0 \rightarrow i) \in \mathbf{E}_{0 i}} V_{i}\right) \times\left(\prod_{(i \rightarrow 0) \in \mathbf{E}_{i 0}} V_{i}^{\vee}\right) \times W \times W^{\vee} .
$$

We have the function $w^{\star}$ defined by

$$
w^{\star}: \operatorname{Rep}\left(Q^{\dagger}\right)_{W} \rightarrow \mathbb{C}, w^{\star}\left(x, u, u^{\prime}, v, v^{\prime}\right)=w\left(x, u, u^{\prime}\right)+\left\langle v, v^{\prime}\right\rangle .
$$

Here $w$ is defined in (3.6). Then $w^{\star}$ is semi-invariant for the character $\tau: \widetilde{G} \rightarrow \mathbb{C}^{*}, \tau\left(\left(g_{1}, g_{2}\right)\right)=g_{1} g_{2}^{-1}$, which appeared in (2.4).

We consider the induced $G$-action on $\operatorname{Rep}\left(Q^{\dagger}\right)_{W}$ and the KN stratifications

$$
\operatorname{Rep}\left(Q^{\dagger}\right)_{W}=\left(\operatorname{Rep}\left(Q^{\dagger}\right)_{W}\right)_{\mathrm{ss}}^{ \pm} \sqcup S_{1}^{ \pm} \sqcup S_{2}^{ \pm} \sqcup \ldots
$$

with respect to linearizations $\mathcal{O}\left(\chi_{0}^{ \pm 1}\right)$, with centers $Z_{\alpha}^{ \pm} \subset S_{\alpha}^{ \pm}$and one parameter subgroups $\lambda_{\alpha}^{ \pm}: \mathbb{C}^{*} \rightarrow T$.

We take

$$
\delta=\varepsilon \cdot \chi_{0} \in M_{\mathbb{R}}^{W}, 0<\varepsilon \ll 1
$$

where $\chi_{0}$ is the character $(3.3)$. We have the associated window subcategories (see Definition 2.4)

$$
\mathcal{C}_{\delta}^{ \pm} \subset D_{\mathbb{C}^{*}}\left(\left[\operatorname{Rep}\left(Q^{\dagger}\right)_{W} / G\right], w^{\star}\right)
$$

with respect to the KN stratifications (3.12).

Proposition 3.5. We have $\mathcal{C}_{\delta}^{-} \subset \mathcal{C}_{\delta}^{+}$. Moreover if $\mathbf{E}_{0 i}=\mathbf{E}_{i 0}$, we have $\mathcal{C}_{\delta}^{-}=\mathcal{C}_{\delta}^{+}$.

Proof. By Lemma 3.4, the proposition follows from Theorem 2.7 and Corollary 2.8 . 
3.6. Complex analytic version. We consider the pull-backs of the following commutative diagram by an analytic open neighborhood $0 \in \mathcal{U} \subset$ $\operatorname{Rep}(Q) / / G$

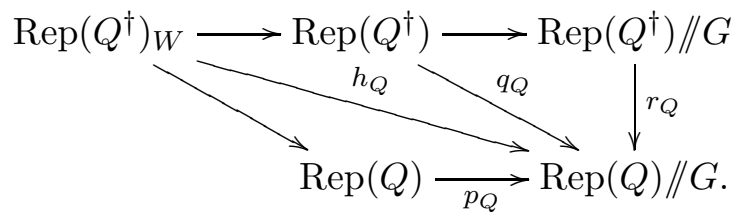

Here each horizontal arrows are either projections or quotient morphisms. Instead of the morphism (3.5), suppose that we are given a $G$-equivariant morphism of analytic vector bundles on $p_{Q}^{-1}(\mathcal{U})$

$$
\vartheta:\left(\prod_{(0 \rightarrow i) \in \mathbf{E}_{0 i}} V_{i}\right) \otimes \mathcal{O}_{p_{Q}^{-1}(\mathcal{U})} \rightarrow\left(\prod_{(i \rightarrow 0) \in \mathbf{E}_{i 0}} V_{i}\right) \otimes \mathcal{O}_{p_{Q}^{-1}(\mathcal{U})} .
$$

Similarly to (3.6), we have the $G$-invariant analytic map

$$
w: q_{Q}^{-1}(\mathcal{U}) \rightarrow \mathbb{C}
$$

which descends to the function $w^{0}$ on $r_{Q}^{-1}(\mathcal{U})$. By pulling back the diagram (3.7) to $r_{Q}^{-1}(\mathcal{U}) \subset \operatorname{Rep}\left(Q^{\dagger}\right) / / G$, we have the diagram

$$
M_{Q^{\dagger}, \mathcal{U}}^{+} \stackrel{\pi_{Q}^{+}}{\longrightarrow} r_{Q}^{-1}(\mathcal{U}) \stackrel{\pi_{Q}^{-}}{\longleftarrow} M_{w^{+}}{ }_{Q^{\dagger}, \mathcal{U}}^{-}
$$

We have the following analytic d-critical loci

$$
M_{\left(Q^{\dagger}, w\right), \mathcal{U}}^{ \pm}=\left\{d w^{ \pm}=0\right\} \subset M_{Q^{\dagger}, \mathcal{U}}^{ \pm} .
$$

Similarly to Proposition 3.2 , we have the following:

Proposition 3.6. Suppose that the followings hold:

$$
\begin{aligned}
& M_{\left(Q^{\dagger}, w\right), \mathcal{U}}^{+} \subset\left[\left(p_{Q}^{-1}(\mathcal{U}) \times \prod_{(0 \rightarrow i) \in \mathbf{E}_{0 i}} V_{i} \times\{0\}\right) / G\right], \\
& M_{\left(Q^{\dagger}, w\right)}^{-} \subset\left[\left(p_{Q}^{-1}(\mathcal{U}) \times\{0\} \times \prod_{(i \rightarrow 0) \in \mathbf{E}_{i 0}} V_{i}^{\vee}\right) / G\right] .
\end{aligned}
$$

Then we have the diagram

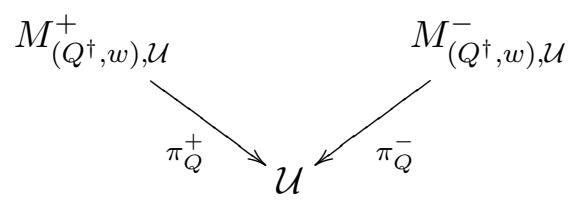


which is an analytic d-critical flip if $\sharp \mathbf{E}_{i 0}<\sharp \mathbf{E}_{0 i}$ for all $i \in V(Q)$. It is an analytic d-critical flop if $\sharp \mathbf{E}_{i 0}=\sharp \mathbf{E}_{0 i}$ for all $i \in V(Q)$.

We have the analytic map

$$
w^{\star}: h_{Q}^{-1}(\mathcal{U}) \rightarrow \mathbb{C}
$$

defined as in (3.11), using (3.16) instead of (3.5). The analytic open subset $h_{Q}^{-1}(\mathcal{U}) \subset \operatorname{Rep}\left(Q^{\dagger}\right)_{W}$ is preserved by the $\widetilde{G}$-action, and the above map $w^{\star}$ is $\tau$ semi-invariant. The derived factorization category $D_{\mathbb{C}^{*}}\left(\left[h_{Q}^{-1}(\mathcal{U}) / G\right], w^{\star}\right)$ and the window subcategories

$$
\mathcal{C}_{\delta, \mathcal{U}}^{ \pm} \subset D_{\mathbb{C}^{*}}\left(\left[h_{Q}^{-1}(\mathcal{U}) / G\right], w^{\star}\right)
$$

are defined similarly to (3.14), using $\delta$ as in (3.13) and KN stratifications (3.12) restricted to $h_{Q}^{-1}(\mathcal{U})$. Then the same argument of Proposition 3.5 shows the following:

Proposition 3.7. We have $\mathcal{C}_{\delta, \mathcal{U}}^{-} \subset \mathcal{C}_{\delta, \mathcal{U}}^{+}$. Moreover if $\mathbf{E}_{0 i}=\mathbf{E}_{i 0}$, we have $\mathcal{C}_{\delta, \mathcal{U}}^{-}=\mathcal{C}_{\delta, \mathcal{U}}^{+}$

\section{Geometry of moduli spaces of Thaddeus pairs}

In this section, we describe Thaddeus pair moduli spaces in terms of critical locus on some smooth stack. Then combined with the main result of [Tod18], we show that the diagram of Thaddus pair moduli space and dual pair moduli space is a d-critical flip (flop), i.e. prove Theorem 1.1 (i).

4.1. Moduli spaces of semistable bundles. Let $C$ be a smooth projective curve over $\mathbb{C}$ with genus $g$. For a vector bundle $E$ on $C$, its slope is defined by

$$
\mu(E):=\frac{\chi(E)}{\operatorname{rank}(E)} \in \mathbb{Q} .
$$

Recall that $E$ is called (semi)stable if for any subbundle $F \subsetneq E$, we have $\mu(F)<(\leq) \mu(E)$.

We denote by $\mathcal{M}(r, d)$ the moduli stack of semistable bundles $E$ on $C$ satisfying the condition

$$
(\operatorname{rank}(E), \chi(E))=(r, d) .
$$

It is well-known that $\mathcal{M}(r, d)$ is written as a global quotient stack, which we will review below. We take $m \gg 0$ and fix $p \in C$. Let $\mathbb{V}$ be a $\mathbb{C}$-vector space such that

$$
\operatorname{dim} \mathbb{V}=d+m r
$$

Let $\operatorname{Quot}(r, d)$ be the Grothendieck quot scheme parameterizing quotients

$$
\phi: \mathbb{V} \otimes \mathcal{O}_{C}(-m p) \rightarrow E
$$


such that $E$ satisfies (4.1). We have an open subset

$$
\mathcal{Q} \subset \operatorname{Quot}(r, d)
$$

corresponding to quotients (4.3) such that $E$ is a semistable bundle and the induced map

$$
H^{0}(\phi(m p)): \mathbb{V} \rightarrow H^{0}(E(m p))
$$

is an isomorphism. Note that both sides of the above map have the same dimension by (4.2). The natural $\mathrm{GL}(\mathbb{V})$-action on $\operatorname{Quot}(r, d)$ preserves the open subset $\mathcal{Q}$, and we have

$$
\mathcal{M}(r, d)=[\mathcal{Q} / \mathrm{GL}(\mathbb{V})] .
$$

Note that $\mathcal{Q}$ is a smooth quasi-projective variety as $\mathcal{M}(r, d)$ is a smooth stack.

For $l \gg m$, let $L_{l}^{\prime}$ be the line bundle on $\operatorname{Quot}(r, d)$ given by

$$
\left.L_{l}^{\prime}\right|_{\left(\mathbb{V} \otimes \mathcal{O}_{C}(-m p) \rightarrow E\right)}=\operatorname{det}\left(H^{0}(C, E(l p))\right) .
$$

The line bundle $L_{l}^{\prime}$ is a GL(V)-linearized ample line bundle. Let $\chi_{0}$ be the determinant character of $\mathrm{GL}(\mathbb{V})$

$$
\chi_{0}: \mathrm{GL}(\mathbb{V}) \rightarrow \mathbb{C}^{*}, g \mapsto \operatorname{det}(g) .
$$

We take a twist of $L_{l}^{\prime}$ by a $\mathrm{GL}(\mathbb{V})$-character to obtain the ample $\mathrm{GL}(\mathbb{V})$ linearized $\mathbb{Q}$-line bundle on $\operatorname{Quot}(r, d)$

$$
L_{l}:=L_{l}^{\prime} \otimes \mathcal{O}\left(-\frac{d+l r}{d+m r} \cdot \chi_{0}\right) .
$$

Then $\mathcal{Q}$ is the semistable locus in the closure $\overline{\mathcal{Q}}$ in $\operatorname{Quot}(r, d)$ with respect to the GL(V)-linearization $L_{l}$ (see [HL97]).

Remark 4.1. The twist in $L_{l}$ is taken so that the $\mathrm{GL}(\mathbb{V})$-linearizion on $L_{l}$ is trivial on the diagonal torus $\mathbb{C}^{*} \subset \mathrm{GL}(\mathbb{V})$. Then the GIT $L_{l}$-stability for the $\mathrm{GL}(\mathbb{V})$-action is equivalent to the $G I T L_{l}^{\prime}$-stability for the $\mathrm{SL}(\mathbb{V})$-action.

By taking the GIT quotient, we obtain the good moduli space for $\mathcal{M}(r, d)$

$$
\iota_{M}: \mathcal{M}(r, d) \rightarrow M(r, d)=\mathcal{Q} / / \mathrm{GL}(\mathbb{V}) .
$$

The GIT quotient $M(r, d)$ is the coarse moduli space of $S$-equivalence classes of semistable bundles on $C$ satisfying (4.1). For a point $p \in M(r, d)$, by taking the associated graded of the Jordan-Hölder filtration, it corresponds to a polystable bundle $E$ of the form

$$
E=\bigoplus_{i=1}^{k} V_{i} \otimes E_{i} .
$$

Here $V_{i}$ is a finite dimensional vector space, each $E_{i}$ is a stable bundle with $\mu\left(E_{i}\right)=\mu(E)$ and $E_{i} \nsucceq E_{j}$ for $i \neq j$. 
4.2. Moduli spaces of Thaddeus pairs. We recall the definition of Thaddeus pairs [Tha94] and introduce the notion of dual Thaddeus pairs. Below we denote by $\operatorname{Bun}(C)$ the category of vector bundles on $C$.

Definition 4.2. (i) A pair

$$
(E, s), E \in \operatorname{Bun}(C), s: \mathcal{O}_{C} \rightarrow E
$$

is called a Thaddeus pair if $E$ is semistable, there is no non-zero subbundle $F \subsetneq E$ with $\mu(F)=\mu(E)$ such that $s$ factors through $F$.

(ii) A pair

$$
\left(E^{\prime}, s^{\prime}\right), E^{\prime} \in \operatorname{Bun}(C), s^{\prime}: E^{\prime} \rightarrow \omega_{C}
$$

is called a dual Thaddeus pair if $E^{\prime}$ is semistable, there is no non-zero subbundle $F^{\prime} \subsetneq E^{\prime}$ with $\mu\left(F^{\prime}\right)=\mu\left(E^{\prime}\right)$ such that $\left.s^{\prime}\right|_{F^{\prime}}=0$.

Lemma 4.3. Giving a dual Thaddeus pair $\left(E^{\prime}, s^{\prime}\right)$ with $\left[E^{\prime}\right] \in \mathcal{M}(r, d)$ is equivalent to giving a Thaddeus pair $(E, s)$ with $[E] \in \mathcal{M}(r,-d)$.

Proof. It is easy to see that the following map gives a desired one to one correspondence

$$
\mathbb{D}:\left(E^{\prime} \stackrel{s^{\prime}}{\rightarrow} \omega_{C}\right) \mapsto\left(\mathcal{O}_{C} \stackrel{s^{\prime \vee}}{\rightarrow} E^{\prime \vee} \otimes \omega_{C}\right) .
$$

We denote by $M^{\mathrm{T}}(r, d)$ the moduli space of Thaddeus pairs $(E, s)$ with $[E] \in \mathcal{M}(r, d)$. The moduli space $M^{\mathrm{T}}(r, d)$ is a smooth projective variety such that

$$
\operatorname{dim} M^{\mathrm{T}}(r, d)=\chi\left(\left(\mathcal{O}_{C} \rightarrow E\right), E\right)=d+r^{2}(g-1) .
$$

Similarly we denote by $M^{\mathrm{DT}}(r, d)$ the moduli space of dual Thaddeus pairs $\left(E^{\prime}, s^{\prime}\right)$ such that $\left[E^{\prime}\right] \in \mathcal{M}(r, d)$. By Lemma 4.3, the correspondence (4.8) gives the isomorphism

$$
\mathbb{D}: M^{\mathrm{DT}}(r, d) \stackrel{\cong}{\rightrightarrows} M^{\mathrm{T}}(r,-d) .
$$

In particular, $M^{\mathrm{DT}}(r, d)$ is a smooth projective variety of dimension $-d+$ $r^{2}(g-1)$. We have the natural morphisms

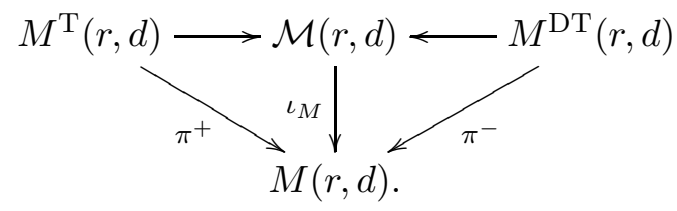

Here $\pi^{ \pm}$are given by $\pi^{+}(E, s)=\iota_{M}(E), \pi^{-}\left(E^{\prime}, s^{\prime}\right)=\iota_{M}\left(E^{\prime}\right)$. 
4.3. GIT descriptions of Thaddeus pair moduli spaces. Let $\mathcal{E}$ be the universal bundle

$$
\mathcal{E} \in \operatorname{Bun}(C \times \mathcal{M}(r, d)) .
$$

Let $\operatorname{pr}_{\mathcal{M}}: C \times \mathcal{M}(r, d) \rightarrow \mathcal{M}(r, d)$ be the projection. As $C$ is a curve, we can write $\operatorname{Rpr}_{\mathcal{M} *} \mathcal{E}$ by a two term complex of vector bundles on $\mathcal{M}(r, d)$

$$
\operatorname{Rpr}_{\mathcal{M} *} \mathcal{E}=\left(\mathcal{F}_{0} \stackrel{\psi}{\rightarrow} \mathcal{F}_{1}\right) .
$$

Here $\mathcal{F}_{0}$ is located in degree zero.

By (4.4), the map $\psi$ is regarded as a GL(V)-equivariant morphism of $\mathrm{GL}(\mathbb{V})$-equivariant vector bundles $\widetilde{\psi}: \widetilde{\mathcal{F}}_{0} \rightarrow \widetilde{\mathcal{F}}_{1}$ on $\mathcal{Q}$. Since the diagonal torus $\mathbb{C}^{*} \subset \mathrm{GL}(\mathbb{V})$ acts on $\mathcal{Q}$ trivially, it acts on fibers of $\widetilde{\mathcal{F}}_{\bullet}$.

Lemma 4.4. We can take a resolution (4.12) so that the diagonal torus $\mathbb{C}^{*}$ acts on fibers of $\widetilde{\mathcal{F}}_{\bullet}$ with weight one.

Proof. We have the decomposition of $\widetilde{\psi}$ into $\mathbb{C}^{*}$-weight spaces

$$
\bigoplus_{n} \widetilde{\psi}_{n}: \bigoplus_{n}\left(\widetilde{\mathcal{F}}_{0}\right)_{n} \rightarrow \bigoplus_{n}\left(\widetilde{\mathcal{F}}_{1}\right)_{n}
$$

where the diagonal torus $\mathbb{C}^{*} \subset \mathrm{GL}(\mathbb{V})$ acts on $\left(\widetilde{\mathcal{F}}_{\bullet}\right)_{n}$ with weight $n$. Since the diagonal $\mathbb{C}^{*}$-actions on $\left.R^{i} \operatorname{pr}_{\mathcal{M} *} \mathcal{E}\right|_{[E]}=H^{i}(C, E)$ are given by the scaling action for $\mathbb{C}^{*} \subset \operatorname{Aut}(E)$, they have weight one. Therefore the morphism $\widetilde{\psi}_{n}$ is an isomorphism for all $n \neq 1$. Then we can replace the RHS of (4.12) by a quasi-isomorphism so that $\left(\widetilde{\mathcal{F}}_{\bullet}\right)_{n}=0$ for all $n \neq 1$.

Below, we take a resolution (4.12) as in Lemma4.4, By setting $\mathcal{F}^{-i}=\mathcal{F}_{i}^{\vee}$, the Grothendieck duality implies

$$
\operatorname{Rpr}_{\mathcal{M} *}\left(\mathcal{E}^{\vee} \otimes \omega_{C}\right) \cong\left(\mathbf{R p r}_{\mathcal{M} *} \mathcal{E}\right)^{\vee}[-1]=\left(\mathcal{F}^{-1} \stackrel{\psi^{\vee}}{\rightarrow} \mathcal{F}^{0}\right) .
$$

Here $\mathcal{F}^{-1}$ is located in degree zero. The map $\psi^{\vee}$ is regarded as a $\operatorname{GL}(\mathbb{V})$ equivariant morphism $\left(\widetilde{\mathcal{F}}^{-1} \stackrel{\widetilde{\psi}^{\vee}}{\rightarrow} \widetilde{\mathcal{F}}^{0}\right)$ on $\mathcal{Q}$, where for $\widetilde{\mathcal{F}}^{-i}=\left(\widetilde{\mathcal{F}}_{i}\right)^{\vee}$. By regarding $\mathcal{F}_{\bullet}, \mathcal{F}^{\bullet}$ as total spaces of vector bundles on $\mathcal{M}(r, d)$, we have the following diagrams of stacks over $\mathcal{M}(r, d)$

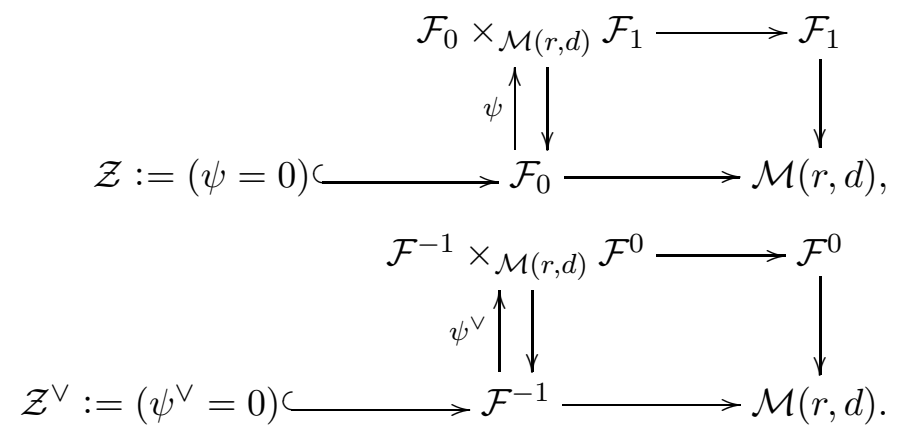


In the above diagrams, $\psi$ and $\psi^{\vee}$ are regarded as sections of vector bundles on $\mathcal{F}_{0}$ and $\mathcal{F}^{-1}$. Let $\mathcal{F}_{0}^{*} \subset \mathcal{F}_{0}, \mathcal{F}^{-1 *} \subset \mathcal{F}^{-1}$ be the complement of the zero sections of the projections $\mathcal{F}_{0} \rightarrow \mathcal{M}(r, d), \mathcal{F}^{-1} \rightarrow \mathcal{M}(r, d)$ respectively.

Lemma 4.5. (i) The stack $\mathcal{Z}$ is isomorphic to the stack of pairs $(E, s)$ for $[E] \in \mathcal{M}(r, d)$ and a morphism $s: \mathcal{O}_{C} \rightarrow E$. Its open subset $\mathcal{Z} \cap \mathcal{F}_{0}^{*}$ is smooth such that

$$
\operatorname{dim}\left(\mathcal{Z} \cap \mathcal{F}_{0}^{*}\right)=\operatorname{dim} M^{\mathrm{T}}(r, d)=d+r^{2}(g-1) .
$$

(ii) The stack $\mathcal{Z}^{\vee}$ is isomorphic to the stack of pairs $\left(E^{\prime}, s^{\prime}\right)$ for $\left[E^{\prime}\right] \in$ $\mathcal{M}(r, d)$ and a morphism $s^{\prime}: E^{\prime} \rightarrow \omega_{C}$. Its open subset $\mathcal{Z}^{\vee} \cap \mathcal{F}^{-1 *}$ is smooth such that

$$
\operatorname{dim}\left(\mathcal{Z}^{\vee} \cap \mathcal{F}^{-1 *}\right)=\operatorname{dim} M^{\mathrm{DT}}(r, d)=-d+r^{2}(g-1) .
$$

Proof. By noting that $\operatorname{Ker}(\psi)=\operatorname{pr}_{\mathcal{M} *} \mathcal{E}$ and $\operatorname{Ker}\left(\psi^{\vee}\right)=\operatorname{pr}_{\mathcal{M} *}\left(\mathcal{E}^{\vee} \otimes \omega_{C}\right)$, the first statements in (i), (ii) are straightforward to prove. As for the second statements, we only show (i) as (ii) is similar.

By the first statement, the stack $\mathcal{Z} \cap \mathcal{F}_{0}^{*}$ is the stack of pairs $(E, s)$ for $[E] \in \mathcal{M}(r, d)$ and $s: \mathcal{O}_{C} \rightarrow E$ is a non-zero morphism. By the deformationobstruction theory of pairs, it is enough to show the vanishing

$$
\mathcal{H}^{1}\left(\mathbf{R} \Gamma\left(\left(\mathcal{O}_{C} \rightarrow E\right), E\right)\right)=0
$$

where $\mathcal{O}_{C}$ is located in degree zero. By applying $\mathbf{R H o m}(-, E)$ to the distinguished triangle

$$
E[-1] \rightarrow\left(\mathcal{O}_{C} \rightarrow E\right) \rightarrow \mathcal{O}_{C}
$$

we obtain the exact sequence

$$
\operatorname{Ext}^{1}(E, E) \rightarrow H^{1}(C, E) \rightarrow \mathcal{H}^{1}\left(\mathbf{R} \Gamma\left(\left(\mathcal{O}_{C} \rightarrow E\right), E\right)\right) \rightarrow 0 .
$$

The first arrow is Serre dual to the map

$$
\operatorname{Hom}\left(E, \omega_{C}\right) \rightarrow \operatorname{Hom}\left(E, E \otimes \omega_{C}\right)
$$

induced by the map $s: \mathcal{O}_{C} \rightarrow E$. As $s$ is non-zero, the above map is injective, so the first arrow in (4.15) is surjective. Therefore (4.14) holds.

By defining $\widetilde{\mathcal{Z}}, \widetilde{\mathcal{Z}}^{\vee}$ similarly as
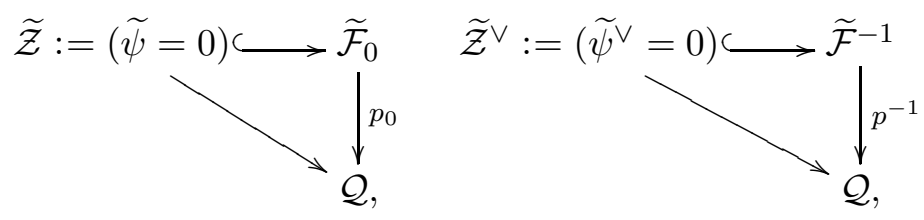

the stacks $\mathcal{Z}, \mathcal{Z}^{\vee}$ are written as

$$
\mathcal{Z}=[\widetilde{\mathcal{Z}} / \mathrm{GL}(\mathbb{V})], \quad \mathcal{Z}^{\vee}=\left[\widetilde{\mathcal{Z}}^{\vee} / \mathrm{GL}(\mathbb{V})\right] .
$$


Note that $\widetilde{\mathcal{Z}}, \widetilde{\mathcal{Z}}^{\vee}$ parametrize diagrams

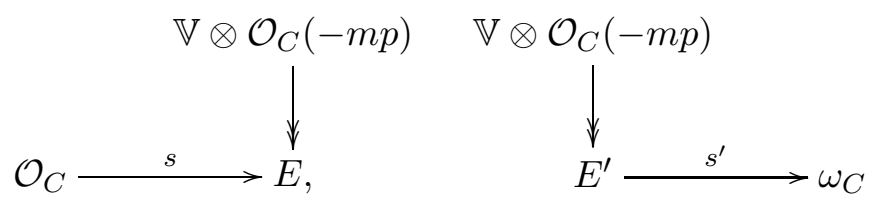

such that $[E] \in \mathcal{M}(r, d),\left[E^{\prime}\right] \in \mathcal{M}(r, d)$ respectively. In the diagram (4.16), we define the following GL( $\mathbb{V})$-equivariant $\mathbb{Q}$-line bundles

$$
\begin{aligned}
& L_{l}^{+}=p_{0}^{*} L_{l} \otimes \mathcal{O}\left(\chi_{0}^{\varepsilon}\right) \in \operatorname{Pic}_{\mathrm{GL}(\mathbb{V})}\left(\widetilde{\mathcal{F}}_{0}\right)_{\mathbb{Q}}, \\
& L_{l}^{-}=p^{-1 *} L_{l} \otimes \mathcal{O}\left(\chi_{0}^{-\varepsilon}\right) \in \operatorname{Pic}_{\mathrm{GL}(\mathbb{V})}\left(\widetilde{\mathcal{F}}^{-1}\right)_{\mathbb{Q}}
\end{aligned}
$$

for a rational number $0<\varepsilon \ll 1$.

Lemma 4.6. (i) A left diagram of (4.17) is $L_{l}^{+}$-semistable if and only if the pair $\left(\mathcal{O}_{C} \stackrel{s}{\rightarrow} E\right)$ is a Thaddeus pair.

(ii) A right diagram of (4.17) is $L_{l}^{-}$-semistable if and only if the pair $\left(E^{\prime} \stackrel{s^{\prime}}{\rightarrow} \omega_{C}\right)$ is a dual Thaddeus pair.

Proof. The lemma follows from a standard argument applying the HilbertMumford criterion. See [ST11, Tod14, Lin18] for related arguments.

By the above lemma, we have the following descriptions of Thaddeus pair moduli spaces

$$
\begin{aligned}
& \left(\mathcal{F}_{0}\right)_{L_{l}^{+} \text {-ss }} \cap \mathcal{Z}=\mathcal{Z}_{L_{l}^{+} \text {-ss }}=M^{\mathrm{T}}(r, d), \\
& \left(\mathcal{F}^{-1}\right)_{L_{l}^{-} \text {-ss }} \cap \mathcal{Z}^{\vee}=\mathcal{Z}_{L_{l}^{-} \text {-ss }}^{\vee}=M^{\mathrm{DT}}(r, d) .
\end{aligned}
$$

4.4. Descriptions by critical locus. Let $\widetilde{\mathcal{Y}}$ be the variety defined by

$$
\widetilde{\mathcal{Y}}:=\widetilde{\mathcal{F}}_{0} \times_{\mathcal{Q}} \widetilde{\mathcal{F}}^{-1}
$$

and define the stack $\mathcal{Y}$ to be

$$
\mathcal{Y}:=\mathcal{F}_{0} \times_{\mathcal{M}(r, d)} \mathcal{F}^{-1}=[\widetilde{\mathcal{Y}} / \mathrm{GL}(\mathbb{V})] .
$$

We define the function $w$ on $\widetilde{\mathcal{Y}}$ to be

$$
w: \widetilde{\mathcal{F}}_{0} \times_{\mathcal{Q}} \widetilde{\mathcal{F}}^{-1} \rightarrow \mathbb{A}^{1},\left(x, u, u^{\prime}\right) \mapsto\left\langle\left.\widetilde{\psi}\right|_{x}(u), u^{\prime}\right\rangle .
$$

Here $x \in \mathcal{Q},\left.u \in \widetilde{\mathcal{F}}_{0}\right|_{x}$ and $\left.u^{\prime} \in \widetilde{\mathcal{F}}^{-1}\right|_{x}$. Then $w$ is $\operatorname{GL}(\mathbb{V})$-invariant, so it descends to the morphism

$$
w: \mathcal{Y} \rightarrow \mathbb{A}^{1}
$$

We define $\widetilde{\mathcal{W}}, \mathcal{W}$ to be

$$
\widetilde{\mathcal{W}}:=\{d w=0\} \subset \widetilde{\mathcal{Y}}, \mathcal{W}:=[\widetilde{\mathcal{W}} / \mathrm{GL}(\mathbb{V})]=\{d w=0\} \subset \mathcal{Y} .
$$


Let $p_{\mathcal{Y}}: \widetilde{\mathcal{Y}} \rightarrow \mathcal{Q}$ be the projection, and define the following GL(V)-linearized ample $\mathbb{Q}$-line bundles on $\widetilde{\mathcal{Y}}$

$$
L_{l}^{ \pm}:=p_{\mathcal{Y}}^{*} L_{l} \otimes \mathcal{O}\left(\chi_{0}^{ \pm \varepsilon}\right), \varepsilon \in \mathbb{Q}, 0<\varepsilon \ll 1 .
$$

The above $\mathbb{Q}$-line bundles restrict to the $\mathbb{Q}$-line bundles $(4.18$ ) by the closed immersions

$$
i_{0}: \mathcal{F}_{0} \hookrightarrow \mathcal{Y}, i^{-1}: \mathcal{F}^{-1} \hookrightarrow \mathcal{Y}
$$

given by zero sections of the projections $\mathcal{Y} \rightarrow \mathcal{F}_{0}, \mathcal{Y} \rightarrow \mathcal{F}^{-1}$ respectively. By (4.19), we have the embeddings

$$
\begin{aligned}
& M^{\mathrm{T}}(r, d)=\mathcal{Z}_{L_{l}^{+} \text {-ss }} \subset \mathcal{F}_{0} \stackrel{i_{0}}{\hookrightarrow} \mathcal{Y}, \\
& M^{\mathrm{DT}}(r, d)=\mathcal{Z}_{L_{l}^{-} \text {-ss }}^{\vee} \subset \mathcal{F}^{-1} \stackrel{i^{-1}}{\hookrightarrow} \mathcal{Y} .
\end{aligned}
$$

Proposition 4.7. The embeddings 4.21) induce the isomorphisms

$$
M^{\mathrm{T}}(r, d) \stackrel{\cong}{\rightrightarrows} \mathcal{W}_{L_{l}^{+} \text {-ss }}, M^{\mathrm{DT}}(r, d) \stackrel{\cong}{\rightrightarrows} \mathcal{W}_{L_{l}^{-} \text {-ss }} .
$$

Proof. We only show the first isomorphism as the latter is similarly proved. By the definition of $w$, the image of $\mathcal{Z} \subset \mathcal{F}_{0} \stackrel{i_{0}}{\hookrightarrow} \mathcal{Y}$ lies in the critical locus $\mathcal{W} \subset \mathcal{Y}$. As $\mathcal{Z} \subset \mathcal{W}$ is a closed immersion, we have

$$
M^{\mathrm{T}}(r, d)=\mathcal{Z}_{L_{l}^{+} \text {-ss }} \hookrightarrow \mathcal{W}_{L_{l}^{+} \text {-ss }} .
$$

It is enough to show that $\mathcal{W}_{L_{l}^{+} \text {-ss }} \subset \mathcal{Z}$, as if this is the case we have

$$
\mathcal{W}_{L_{l}^{+} \text {-ss }} \subset \mathcal{Z}_{L_{l}^{+} \text {-ss }}=M^{\mathrm{T}}(r, d) .
$$

Below we show that $\mathcal{W}_{L_{l}^{+} \text {-ss }} \subset \mathcal{Z}$. By the $L_{l}^{+}$-stability, we have

$$
\mathcal{W}_{L_{l}^{+} \text {-ss }} \subset\left(\mathcal{F}_{0}^{*} \times_{\mathcal{M}(r, d)} \mathcal{F}^{-1}\right) \cap \mathcal{W}
$$

Indeed for the anti-diagonal $\lambda: \mathbb{C}^{*} \rightarrow \mathrm{GL}(\mathbb{V})$ and a point $x \in \widetilde{\mathcal{F}}^{-1} \stackrel{i^{-1}}{\hookrightarrow} \widetilde{\mathcal{Y}}$, as $\lambda$ acts on $\widetilde{\mathcal{F}}^{-1}$ with weight one, we have

$$
y:=\lim _{t \rightarrow 0} \lambda(t)(x) \in \mathcal{Q} \subset \widetilde{\mathcal{Y}}
$$

where the latter is the zero section of the projection $\widetilde{\mathcal{Y}} \rightarrow \mathcal{Q}$. Then we have

$$
\text { weight }_{\lambda}\left(\left.L_{l}^{+}\right|_{y}\right)=\varepsilon \cdot\left\langle\lambda, \chi_{0}\right\rangle=-\varepsilon \cdot \operatorname{dim} \mathbb{V}<0 .
$$

Therefore $x$ is not $L_{l}^{+}$-semistable, and the inclusion (4.22) holds.

By Lemma 4.5, the map

$$
\psi: \mathcal{F}_{0}^{*} \rightarrow \mathcal{F}_{0}^{*} \times \mathcal{M}(r, d) \mathcal{F}_{1}
$$

is a regular section of the vector bundle $\mathcal{F}_{0}^{*} \times{ }_{\mathcal{M}(r, d)} \mathcal{F}_{1} \rightarrow \mathcal{F}_{0}^{*}$, whose zero locus is smooth. Therefore by Lemma 2.2 , we have

$$
\left(\mathcal{F}_{0}^{*} \times_{\mathcal{M}(r, d)} \mathcal{F}^{-1}\right) \cap \mathcal{W}=\mathcal{Z} \cap \mathcal{F}_{0}^{*} \subset \mathcal{Z} .
$$


4.5. Analytic local descriptions. For a point $p \in M(r, d)$, corresponding to a polystable bundle $E$ as in (4.7), we have the associated Ext-quiver $Q_{E}$. Its vertex set is given by $\{1,2, \ldots, k\}$, and the number of edges from $i$ to $j$ is

$$
\sharp(i \rightarrow j)=\operatorname{dim} \operatorname{Ext}^{1}\left(E_{i}, E_{j}\right)= \begin{cases}(g-1) r_{i} r_{j}, & i \neq j, \\ (g-1) r_{i}^{2}+1, & i=j .\end{cases}
$$

Here $r_{i}=\operatorname{rank}\left(E_{i}\right)$. Note that $\sharp(i \rightarrow j)=\sharp(j \rightarrow i)$, i.e. $Q_{E}$ is a symmetric quiver. Then we have

$$
\operatorname{Ext}^{1}(E, E)=\operatorname{Rep}\left(Q_{E}\right):=\prod_{i \rightarrow j} \operatorname{Hom}\left(V_{i}, V_{j}\right)
$$

Let $G_{E}$ be the algebraic group defined by

$$
G_{E}=\operatorname{Aut}(E)=\prod_{i=1}^{k} \mathrm{GL}\left(V_{i}\right) .
$$

The quotient of $\operatorname{Rep}\left(Q_{E}\right)$ by the conjugate action of $G_{E}$ is the moduli stack of $Q_{E}$-representations with dimension vector $\left(\operatorname{dim} V_{i}\right)_{1 \leq i \leq k}$. We have the morphism to the good moduli space

$$
\iota_{Q}:\left[\operatorname{Rep}\left(Q_{E}\right) / G_{E}\right] \rightarrow \operatorname{Rep}\left(Q_{E}\right) / / G_{E} .
$$

The following is a special case of the main result of [Tod18].

Theorem 4.8. ([Tod18]) There exist analytic open neighborhoods $p \in \mathcal{V} \subset$ $M(r, d), 0 \in \mathcal{U} \subset \operatorname{Rep}\left(Q_{E}\right) / / G_{E}$ and commutative isomorphisms

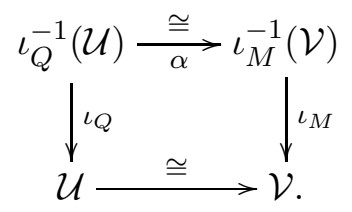

Here the map a sends $0 \in \iota_{Q}^{-1}(\mathcal{U})$ to $[E] \in \iota_{M}^{-1}(\mathcal{V})$.

Let $p_{Q}$ be the quotient morphism

$$
p_{Q}: \operatorname{Rep}\left(Q_{E}\right) \rightarrow \operatorname{Rep}\left(Q_{E}\right) / / G_{E} .
$$

We take $0 \in \mathcal{U}$ to be a Stein analytic open neighborhood, so that $p_{Q}^{-1}(\mathcal{U})$ is also Stein. Then we have the following lemma:

Lemma 4.9. For the isomorphism $\alpha$ in Theorem 4.8, the complex

$$
\left.\alpha^{*}\left(\mathcal{F}_{0} \stackrel{\psi}{\rightarrow} \mathcal{F}_{1}\right)\right|_{\iota_{M}^{-1}(\mathcal{V})}
$$

is isomorphic to the direct sum

$$
\left(H^{0}(E) \otimes \mathcal{O}_{p_{Q}^{-1}(\mathcal{U})} \stackrel{\vartheta}{\rightarrow} H^{1}(E) \otimes \mathcal{O}_{p_{Q}^{-1}(\mathcal{U})}\right) \oplus\left(W \otimes \mathcal{O}_{p_{Q}^{-1}(\mathcal{U})} \stackrel{\text { id }}{\rightarrow} W \otimes \mathcal{O}_{p_{Q}^{-1}(\mathcal{U})}\right)
$$

for some $G_{E}$-equivariant morphism $\vartheta$ and a finite dimensional $G_{E}$-representation $W$. 
Proof. We can write

$$
\left.\alpha^{*}\left(\mathcal{F}_{0} \stackrel{\psi}{\rightarrow} \mathcal{F}_{1}\right)\right|_{\iota_{M}^{-1}(\mathcal{V})} \cong\left(R_{0} \otimes \mathcal{O}_{p_{Q}^{-1}(\mathcal{U})} \stackrel{\phi}{\rightarrow} R_{1} \otimes \mathcal{O}_{p_{Q}^{-1}(\mathcal{U})}\right)
$$

for some finite dimensional $G_{E}$-representations $R_{0}, R_{1}$ and a $G_{E}$-equivariant morphism $\phi$. As the map $\alpha$ sends $0 \in p_{Q}^{-1}(\mathcal{U})$ to $[E] \in \iota_{M}^{-1}(\mathcal{V})$, we have

$$
\operatorname{Ker}\left(\left.\phi\right|_{0}\right)=H^{0}(E), \operatorname{Cok}\left(\left.\phi\right|_{0}\right)=H^{1}(E)
$$

as $G_{E}$-representations. As $G_{E}$ is reductive, we have

$$
R_{0}=H^{0}(E) \oplus W, R_{1}=H^{1}(E) \oplus W
$$

for some finite dimensional $G_{E}$-representation $W$, and the map $\left.\phi\right|_{0}$ is written as

$$
\left(H^{0}(E) \stackrel{0}{\rightarrow} H^{1}(E)\right) \oplus(W \stackrel{\text { id }}{\rightarrow} W) .
$$

Then $\phi$ is written as

$$
\phi=\left(\begin{array}{ll}
\phi_{11} & \phi_{12} \\
\phi_{21} & \phi_{22}
\end{array}\right):\left(H^{0}(E) \oplus W\right) \otimes \mathcal{O}_{p_{Q}^{-1}(\mathcal{U})} \rightarrow\left(H^{1}(E) \oplus W\right) \otimes \mathcal{O}_{p_{Q}^{-1}(\mathcal{U})} .
$$

Here $\phi_{i j}$ are $G_{E}$-equivariant morphisms

$$
\phi_{i j}: U_{i} \otimes \mathcal{O}_{p_{Q}^{-1}(\mathcal{U})} \rightarrow U_{j}^{\prime} \otimes \mathcal{O}_{p_{Q}^{-1}(\mathcal{U})}
$$

for $U_{1}=H^{0}(E), U_{1}^{\prime}=H^{1}(E), U_{2}=U_{2}^{\prime}=W$, such that

$$
\left.\phi_{i j}\right|_{0}=0,(i, j) \neq(2,2),\left.\phi_{22}\right|_{0}=\mathrm{id} .
$$

By shrinking $\mathcal{U}$ if necessary, we may assume that $\phi_{22}$ is an isomorphism. Then by replacing $\phi$ by an automorphism of $W \otimes \mathcal{O}_{p_{Q}^{-1}(\mathcal{U})}$, we may assume that $\phi_{22}=\mathrm{id}$. Then by the following replacement of $\phi$ by automorphisms of both sides

$$
\left(\begin{array}{cc}
\mathrm{id} & -\phi_{12} \\
0 & \mathrm{id}
\end{array}\right)\left(\begin{array}{ll}
\phi_{11} & \phi_{12} \\
\phi_{21} & \phi_{22}
\end{array}\right)\left(\begin{array}{cc}
1 & 0 \\
-\phi_{21} & 1
\end{array}\right)
$$

we may assume that $\phi_{12}=0, \phi_{21}=0$. By setting $\vartheta=\phi_{11}$, we obtain the lemma.

In what follows, we assume that $d \geq 0$. Then we have

$$
h^{0}\left(E_{i}\right)-h^{1}\left(E_{i}\right)=\chi\left(E_{i}\right)=r_{i} \cdot \frac{d}{r} \geq 0
$$

for $1 \leq i \leq k$. For each $1 \leq i \leq k$, let us fix finite subsets and an injective map

$$
\mathbf{E}_{0 i} \subset H^{0}\left(E_{i}\right), \mathbf{E}_{i 0} \subset H^{1}\left(E_{i}\right), \mathbf{E}_{i 0} \hookrightarrow \mathbf{E}_{0 i}
$$


where $\mathbf{E}_{0 i}, \mathbf{E}_{i 0}$ give basis of $H^{0}\left(E_{i}\right), H^{1}\left(E_{i}\right)$ respectively. Let $Q_{E}^{\dagger}$ be the extended quiver as in Section [3.1, constructed from $Q=Q_{E}$ and $\mathbf{E}_{0 i}, \mathbf{E}_{i 0}$ as above. Then we have

$$
H^{0}(E)=\prod_{(0 \rightarrow i) \in \mathbf{E}_{0 i}} V_{i}, \quad H^{1}(E)=\prod_{(0 \rightarrow i) \in \mathbf{E}_{i 0}} V_{i} .
$$

Therefore in the notation of Section 3.1, we have

$$
\begin{aligned}
& \operatorname{Rep}\left(Q_{E}^{\dagger}\right)=\operatorname{Rep}\left(Q_{E}\right) \times H^{0}(E) \times H^{1}(E)^{\vee}, \\
& \operatorname{Rep}\left(Q_{E}^{\dagger}\right)_{W}=\operatorname{Rep}\left(Q_{E}\right) \times H^{0}(E) \times H^{1}(E)^{\vee} \times W \times W^{\vee} .
\end{aligned}
$$

Here $W$ is a $G_{E}$-representation in Lemma 4.9, As in the diagram (3.15), we have the map

$$
h_{Q}: \operatorname{Rep}\left(Q_{E}^{\dagger}\right)_{W} \rightarrow \operatorname{Rep}\left(Q_{E}\right) / / G_{E}
$$

by composing the projection and the quotient map. So we have

$$
\left[h_{Q}^{-1}(\mathcal{U}) / G_{E}\right]=\left[\left(p_{Q}^{-1}(\mathcal{U}) \times H^{0}(E) \times H^{1}(E)^{\vee} \times W \times W^{\vee}\right) / G_{E}\right] .
$$

Let $\pi_{\mathcal{Y}}$ be the composition of the natural maps

$$
\pi_{\mathcal{Y}}: \mathcal{Y} \rightarrow \mathcal{M}(r, d) \rightarrow M(r, d) .
$$

Then Theorem 4.8 together with Lemma 4.9 imply that we have the following commutative isomorphisms

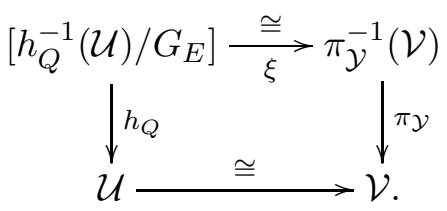

Under the isomorphism $\xi$, the line bundles $L_{l}^{ \pm}$on $\mathcal{Y}$ are pulled back to the $G_{E}$-equivariant $\mathbb{Q}$-line bundles

$$
\mathcal{O}\left(\chi_{0}^{ \pm \varepsilon}\right) \in \operatorname{Pic}_{G_{E}}\left(h_{Q}^{-1}(\mathcal{U})\right)_{\mathbb{Q}}
$$

where $\chi_{0}: G_{E} \rightarrow \mathbb{C}^{*}$ is given by (3.3) for $Q=Q_{E}$. Moreover the composition

$$
w^{\star}:\left[h_{Q}^{-1}(\mathcal{U}) / G_{E}\right] \stackrel{\xi}{\rightarrow} \pi_{\mathcal{Y}}^{-1}(\mathcal{V}) \hookrightarrow \mathcal{Y} \stackrel{w}{\rightarrow} \mathbb{A}^{1}
$$

is given by

$$
w^{\star}\left(x, u, u^{\prime}, v, v^{\prime}\right)=\left\langle\left.\vartheta\right|_{x}(u), u^{\prime}\right\rangle+\left\langle v, v^{\prime}\right\rangle .
$$

Here $x \in p_{Q}^{-1}(\mathcal{U}), u \in H^{0}(E), u^{\prime} \in H^{1}(E)^{\vee}, v \in W, v^{\prime} \in W^{\vee}$, and $\vartheta$ is given in Lemma 4.9. Then we show the following: 
Theorem 4.10. For $d>0$, the diagram

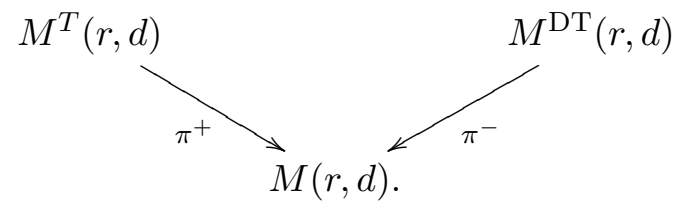

is an analytic d-critical flip. For $d=0$, it is an analytic d-critical flop.

Proof. Let $p \in M(r, d)$ corresponds to a polystable bundle $E$ as in (4.7), and take $p \in \mathcal{V} \subset M(r, d), 0 \in \mathcal{U} \subset \operatorname{Rep}\left(Q_{E}\right) / / G$ as in Theorem 4.8. Then we have the diagram (4.24) as discussed above. The critical locus of the function (4.26) is the same as the critical locus of

$$
w_{Q}:\left[\left(p_{Q}^{-1}(\mathcal{U}) \times H^{0}(E) \times H^{1}(E)^{\vee}\right) / G_{E}\right] \rightarrow \mathbb{C}
$$

defined by $w_{Q}\left(x, u, u^{\prime}\right)=\left\langle\left.\vartheta\right|_{x}(u), u^{\prime}\right\rangle$. Together with the compatibility of linearizations (4.25), in the notation of Section 3.6 we have the commutative isomorphisms

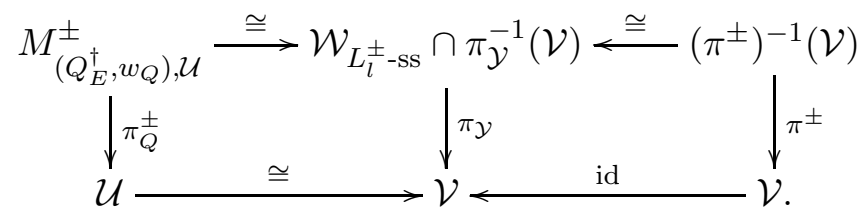

Here the right diagram follows from Proposition 4.7, and

$$
M_{\left(Q_{E}^{\dagger}, w_{Q}\right), \mathcal{U}}^{ \pm}=\left[\left(\left(p_{Q}^{-1}(\mathcal{U}) \times H^{0}(E) \times H^{1}(E)^{\vee}\right)_{\mathcal{O}\left(\chi_{0}^{ \pm \varepsilon}\right)-\mathrm{ss}} \cap\left\{d w_{Q}=0\right\}\right) / G_{E}\right]
$$

as given in (3.17) for $Q^{\dagger}=Q_{E}^{\dagger}$. The above isomorphisms imply that the assumption in Proposition 3.6 is satisfied, so the result follows from Proposition 3.6 .

\section{Derived functors Between Thaddeus pair Moduli spaces}

In this section, we combine the arguments so far and finish the proof of Theorem 1.1 (ii). We also investigate the fully-faithful functor $\Phi_{M}$ on the stable locus, and give an explicit description of the kernel object.

5.1. Window subcategories for Thaddeus pair moduli spaces. Let $\widetilde{\mathrm{GL}}(\mathbb{V})$ be the algebraic group defined in (2.3) for $G=\mathrm{GL}(\mathbb{V})$ and the diagonal torus $\mathbb{C}^{*} \subset \mathrm{GL}(\mathbb{V})$. Then $\widetilde{\mathrm{GL}}(\mathbb{V})$ acts on $\widetilde{\mathcal{Y}}$ by

$$
\begin{aligned}
\left(g_{1}, g_{2}\right) \cdot\left(x, u, u^{\prime}\right) & =\left(g_{1}(x), g_{1}(u), g_{2}\left(u^{\prime}\right)\right) \\
& =\left(g_{2}(x), g_{1}(u), g_{2}\left(u^{\prime}\right)\right)
\end{aligned}
$$

for $\left(g_{1}, g_{2}\right) \in \widetilde{\mathrm{GL}}(\mathbb{V}), x \in \mathcal{Q},\left.u \in \widetilde{\mathcal{F}}_{0}\right|_{x}$ and $\left.u^{\prime} \in \widetilde{\mathcal{F}}^{-1}\right|_{x}$. Here the latter equality holds as the diagonal torus $\mathbb{C}^{*} \subset \mathrm{GL}(\mathbb{V})$ acts on $\mathcal{Q}$ trivially. 
The function $w$ in (4.20) is $\tau$ semi-invariant, where $\tau: \widetilde{\mathrm{GL}}(\mathbb{V}) \rightarrow \mathbb{C}^{*}$ is the character $\left(g_{1}, g_{2}\right) \mapsto g_{1} g_{2}^{-1}$ in the exact sequence (2.4).

We have the KN stratifications

$$
\widetilde{\mathcal{Y}}=\widetilde{\mathcal{Y}}_{L_{l}^{ \pm} \text {-ss }} \sqcup S_{1}^{ \pm} \sqcup S_{2}^{ \pm} \sqcup \cdots, \mathcal{Y}=\mathcal{Y}_{L_{l}^{ \pm} \text {-ss }} \sqcup \mathcal{S}_{1}^{ \pm} \sqcup \mathcal{S}_{2}^{ \pm} \sqcup \cdots
$$

with respect to the GL(V)-linearizations $L_{l}^{ \pm}$, and the associated window subcategories (see Definition 2.4)

$$
\mathcal{C}_{\mathcal{Y}}^{ \pm} \subset D_{\mathbb{C}^{*}}(\mathcal{Y}, w)
$$

Proposition 5.1. We have equivalences of triangulated categories

$$
\mathcal{C}_{\mathcal{Y}}^{+} \stackrel{\sim}{\rightarrow} D^{b}\left(M^{\mathrm{T}}(r, d)\right), \mathcal{C}_{\mathcal{Y}}^{-} \stackrel{\sim}{\rightarrow} D^{b}\left(M^{\mathrm{DT}}(r, d)\right)
$$

Proof. By Theorem 2.5 and its generalization [HLc], the following compositions are equivalences

$$
\mathcal{C}_{\mathcal{Y}}^{ \pm} \subset D_{\mathbb{C}^{*}}(\mathcal{Y}, w) \stackrel{\text { res }}{\rightarrow} D_{\mathbb{C}^{*}}\left(\mathcal{Y}_{L_{l}^{ \pm} \text {-ss }}, w\right)
$$

On the other hand, we have the open immersion

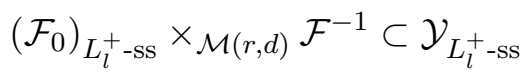

by the definition of GIT stability. By Proposition 4.7 the critical locus of $w$ restricted to $\mathcal{Y}_{L_{l}^{+} \text {-ss }}$ is $\mathcal{Z}_{L_{l}^{+} \text {-ss }}$, which is contained in $\left(\mathcal{F}_{0}\right)_{L_{l}^{+} \text {-ss }} \times_{\mathcal{M}(r, d)} \mathcal{F}^{-1}$. Therefore the restriction functor

$$
\text { res: } D_{\mathbb{C}^{*}}\left(\mathcal{Y}_{L_{l}^{+} \text {-ss }}, w\right) \rightarrow D_{\mathbb{C}^{*}}\left(\left(\mathcal{F}_{0}\right)_{L_{l}^{+} \text {-ss }} \times_{\mathcal{M}(r, d)} \mathcal{F}^{-1}, w\right)
$$

is an equivalence (see [HLS, Lemma 5.5]). Then using the splitting $\gamma_{0}$ in (2.5), a version of Knörrer periodicity in Theorem 2.3 implies the equivalence

$$
D^{b}\left(M^{\mathrm{T}}(r, d)\right)=D^{b}\left(\mathcal{Z}_{L_{l}^{+} \text {-ss }}\right) \stackrel{\sim}{\rightarrow} D_{\mathbb{C}^{*}}\left(\left(\mathcal{F}_{0}\right)_{L_{l}^{+} \text {-ss }} \times_{\mathcal{M}(r, d)} \mathcal{F}^{-1}, w\right) .
$$

Therefore we obtain the first equivalence of (5.2). The second equivalence of (5.2) also holds by the same argument, using the splitting $\gamma_{1}$ in (2.5) instead of $\gamma_{0}$.

Now Theorem 1.1 follows from the following theorem together with Proposition 5.1 and the isomorphism (4.10).

Theorem 5.2. For $d>0$, we have $\mathcal{C}_{\mathcal{Y}}^{-} \subset \mathcal{C}_{\mathcal{Y}}^{+}$. For $d=0$, we have $\mathcal{C}_{\mathcal{Y}}^{-}=\mathcal{C}_{\mathcal{Y}}^{+}$.

Proof. Let us take $p \in \mathcal{V} \subset M(r, d)$ and consider the diagram (4.24) as before. Then by the compatibility of linearizations (4.25), the KN stratifications (5.1) restricted to $\pi_{\mathcal{Y}}^{-1}(\mathcal{V})$ coincides with the KN stratifications (3.12) for $Q^{\dagger}=Q_{E}^{\dagger}$ restricted to $\left[h_{Q}^{-1}(\mathcal{U}) / G_{E}\right]$ under the isomorphism $\xi$. Therefore for an object $\mathcal{E} \in D_{\mathbb{C}^{*}}(\mathcal{Y}, w)$, it is an object in $\mathcal{C}_{\mathcal{Y}}^{ \pm}$if and only if for any point $p \in M(r, d)$ and an analytic open neighborhood $p \in \mathcal{V} \subset M(r, d)$, we have

$$
\xi^{*}\left(\left.\mathcal{E}\right|_{\pi_{\mathcal{Y}}^{-1}(\mathcal{V})}\right) \in \mathcal{C}_{\delta, \mathcal{U}}^{ \pm} \subset D_{\mathbb{C}^{*}}\left(\left[h_{Q}^{-1}(\mathcal{U}) / G_{E}\right], w^{\star}\right)
$$


where $w^{\star}$ is given by (4.26) and $\mathcal{C}_{\delta, \mathcal{U}}^{ \pm}$are the window subcategories (3.18) for the quiver $Q^{\dagger}=Q_{E}^{\dagger}$. Since $\mathcal{C}_{\delta, \mathcal{U}}^{-} \subset \mathcal{C}_{\delta, \mathcal{U}}^{+}$by Proposition 3.7, we conclude $\mathcal{C}_{\mathcal{Y}}^{-} \subset \mathcal{C}_{\mathcal{Y}}^{+}$for $d>0$. Similarly we have $\mathcal{C}_{\mathcal{Y}}^{-}=\mathcal{C}_{\mathcal{Y}}^{+}$for $d=0$.

5.2. Geometry on the stable locus. In what follows, we will describe the explicit form of the fully faithful functor constructed in Theorem 1.1 on the stable locus.

Let $p \in M(r, d)$ be a point corresponding to a stable vector bundle $E$, $Q=Q_{E}$ the associated Ext-quiver, and $G_{E}=T=\mathbb{C}^{*}$. Note that $Q$ has only one vertex with $c$-loops for $c:=\operatorname{ext}^{1}(E, E)$. Let $W$ be a $\mathbb{C}^{*}$-representation appeared in Lemma 4.9, which is of weight one by Lemma 4.4. Hence we have

$$
\operatorname{Rep}\left(Q^{\dagger}\right)_{W}=U \times V^{+} \times V^{-},
$$

where $U, V^{+}, V^{-}$are $\mathbb{C}^{*}$-representations of weights $0,+1,-1$, respectively, given by

$$
U:=\operatorname{Ext}^{1}(E, E), V^{+}:=H^{0}(E) \times W, V^{-}:=H^{1}(E) \times W^{\vee} .
$$

Below we set $a:=\operatorname{rk} \mathcal{F}_{0}=\operatorname{dim} V^{+}, b:=\operatorname{rk} \mathcal{F}_{1}=\operatorname{dim} V^{-}$, and assume that $a \geq b$, which is equivalent to $d \geq 0$.

We take analytic open neighborhoods

$$
p \in \mathcal{V} \subset M(r, d), \quad 0 \in \mathcal{U} \subset \operatorname{Rep}(Q) / / G=U
$$

as in Theorem 4.8, Then we have

$$
\left[h_{Q}^{-1}(\mathcal{U})_{\mathrm{ss}}^{ \pm} / G\right]=Y_{\mathcal{U}}^{ \pm} \subset\left[h_{Q}^{-1}(\mathcal{U}) / G\right]=\left[\left(V^{+} \times V^{-}\right) \mathcal{U} / \mathbb{C}^{*}\right]
$$

where $h_{Q}^{-1}(\mathcal{U})_{\mathrm{ss}}^{ \pm} \subset h_{Q}^{-1}(\mathcal{U})$ are semistable locus with respect to the characters $\pm: \mathbb{C}^{*} \rightarrow \mathbb{C}^{*},(-) \mathcal{U}$ means $(-) \times \mathcal{U}$ and

$$
\begin{aligned}
& Y^{+}:=\operatorname{Tot}_{\mathbb{P}\left(V^{+}\right)}\left(\mathcal{O}_{\mathbb{P}\left(V^{+}\right)}(-1) \otimes V^{-}\right), \\
& Y^{-}:=\operatorname{Tot}_{\mathbb{P}\left(V^{-}\right)}\left(\mathcal{O}_{\mathbb{P}\left(V^{-}\right)}(-1) \otimes V^{+}\right) .
\end{aligned}
$$

The function $w^{\star}$ on $h_{Q}^{-1}(\mathcal{U})$ given in (4.26) is written as

$$
w^{\star}=\sum_{1 \leq i \leq 1,1 \leq j \leq b} x_{i} y_{j} w_{i j}(\vec{u})
$$

where $\vec{x}, \vec{y}$ are coordinates of $V^{+}, V^{-}$, and $w_{i j}(\vec{u}) \in \Gamma\left(\mathcal{O}_{\mathcal{U}}\right)$.

We also set

$$
Z:=\left(V^{+} \times V^{-}\right) / / \mathbb{C}^{*}=\operatorname{Spec} \mathbb{C}\left[x_{i} y_{j}: 1 \leq i \leq a, 1 \leq i \leq b\right] .
$$

Note that $Y^{ \pm}, Z$ are GIT quotients of $V^{+} \times V^{-}$by the above $\mathbb{C}^{*}$-action, and we have the standard toric flip diagram

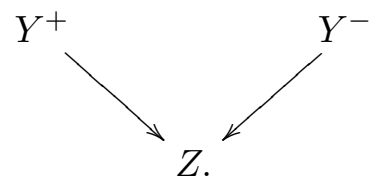


5.3. Descriptions of the kernel object: local case. In the case of the previous subsection, a pair of the KN stratifications (3.12) is an elementary wall crossing in the sense of [BFK19, Definition 3.5.1], and we can describe the corresponding window subcategories

$$
\mathcal{C}_{\delta, \mathcal{U}}^{ \pm} \subset D_{\mathbb{C}^{*}}\left(\left[h_{Q}^{-1}(\mathcal{U}) / \mathbb{C}^{*}\right], w^{\star}\right)
$$

in the following way: For an interval $I \subset \mathbb{R}$, define the subcategory $\mathcal{W}_{I} \subset$ $D_{\mathbb{C}^{*}}\left(\left[h_{Q}^{-1}(\mathcal{U}) / \mathbb{C}^{*}\right], w^{\star}\right)$ to be the triangulated subcategory generated by the factorizations (2.7) where $\mathcal{P}_{j}$ are of the form

$$
\mathcal{P}_{j}=\bigoplus_{i \in I \cap \mathbb{Z}} \mathcal{O}_{h_{Q}^{-1}(\mathcal{U})}(i)^{\oplus l_{i, j}}, \quad j=0,1, \quad l_{i, j} \in \mathbb{Z}_{\geq 0}
$$

as $\mathbb{C}^{*}$-equivariant sheaves. Then we have $\mathcal{C}_{\delta, \mathcal{U}}^{ \pm}=\mathcal{W}_{I^{ \pm}}$, where the intervals $I^{ \pm} \subset \mathbb{R}$ are defined as follows.

$$
I^{-}:=\left\lceil-\left\lceil\frac{b}{2}\right\rceil+1,\left\lfloor\frac{b}{2}\right\rfloor+1\right), \quad I^{+}:=\left\lceil-\left\lceil\frac{a}{2}\right\rceil+1,\left\lfloor\frac{a}{2}\right\rfloor+1\right) .
$$

As we assumed $a \geq b$, we have $\mathcal{W}_{I^{-}} \subset \mathcal{W}_{I^{+}}$. Let us consider the fully faithful functor $\Psi: D_{\mathbb{C}^{*}}\left(Y_{\mathcal{U}}^{-}, w^{\star}\right) \rightarrow D_{\mathbb{C}^{*}}\left(Y_{\mathcal{U}}^{+}, w^{\star}\right)$ which fits into the following commutative diagram:

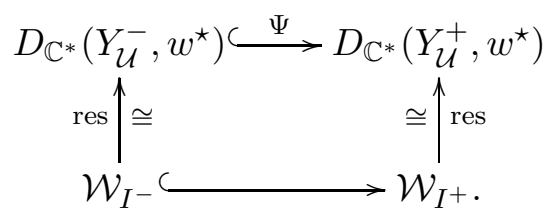

First we will describe the semi-orthogonal complement of the functor $\Psi$. Let

$$
g: \mathbb{P}\left(V^{+}\right)_{\mathcal{U}} \rightarrow \mathcal{U}, \quad \iota^{+}: \mathbb{P}\left(V^{+}\right)_{\mathcal{U}} \hookrightarrow Y_{\mathcal{U}}^{+}
$$

be the projection, the inclusion as the zero section, respectively. For an integer $i \in \mathbb{Z}$, define the functor $\Upsilon^{i}$ as

$$
\Upsilon^{i}:=\left(-\otimes \mathcal{O}_{Y_{\mathcal{U}}^{+}}(i)\right) \circ \iota_{*}^{+} g^{*}: D_{\mathbb{C}^{*}}(\mathcal{U}, 0) \rightarrow D_{\mathbb{C}^{*}}\left(Y_{\mathcal{U}}^{+}, w^{\star}\right) .
$$

Then we have the following result due to [HL15, BFK19].

Theorem 5.3 ([HL15, BFK19]). For each integer $i \in \mathbb{Z}$, the functor $\Upsilon^{i}$ is fully faithful. Furthermore, we have the semi-orthogonal decomposition

$$
D_{\mathbb{C}^{*}}\left(Y_{\mathcal{U}}^{+}, w^{\star}\right)=\left\langle\Upsilon^{-\left\lfloor\frac{a}{2}\right\rfloor}, \cdots, \Upsilon^{-\left\lfloor\frac{b}{2}\right\rfloor-1}, \Psi, \Upsilon^{-\left\lfloor\frac{b}{2}\right\rfloor}, \cdots, \Upsilon^{\left\lceil\frac{a}{2}\right\rceil-b-1}\right\rangle .
$$

Next we will determine the kernel of the functor $\Psi$. For this purpose, we set $W:=Y^{+} \times_{Z} Y^{-}$in the diagram (5.4), and $q^{ \pm}: W \rightarrow Y^{ \pm}$be the projections. We have the Fourier-Mukai functor

$$
\Phi^{\mathcal{O}_{W_{\mathcal{U}}}}=\mathbf{R} q_{*}^{+} \mathbf{L} q^{-*}: D_{\mathbb{C}^{*}}\left(Y_{\mathcal{U}}^{-}, w^{\star}\right) \rightarrow D_{\mathbb{C}^{*}}\left(Y_{\mathcal{U}}^{+}, w^{\star}\right) .
$$

Now we prove the following. 
Proposition 5.4. We have an isomorphism of functors

$$
\Psi \cong\left(-\otimes \mathcal{O}_{Y_{\mathcal{U}}^{+}}\left(-\left\lceil\frac{b}{2}\right\rceil+1\right)\right) \circ \Phi^{\mathcal{O}_{W_{\mathcal{U}}} \circ}\left(-\otimes \mathcal{O}_{Y_{\mathcal{U}}^{-}}\left(-\left\lceil\frac{b}{2}\right\rceil+1\right)\right) .
$$

Proof. A version of this result is proved for example in CIJS15, Proposition 5.3]. For the readers' convenience, we include the proof here. We have the following commutative diagram

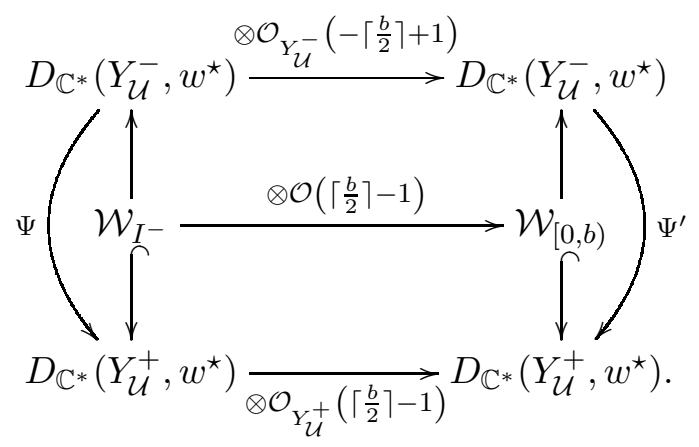

We set $\Phi:=\Phi^{\mathcal{O}_{W \mathcal{U}}}$ and show an isomorphism $\Psi^{\prime} \cong \Phi$ of functors. Take a factorization

$$
E=\left(\mathcal{P}_{0} \stackrel{f}{\rightarrow} \mathcal{P}_{1} \stackrel{g}{\rightarrow} \mathcal{P}_{0}(1)\right) \in \mathcal{W}_{[0, b)},
$$

with

$$
\mathcal{P}_{0}=\bigoplus_{i=1}^{k} \mathcal{O}\left(m_{i}\right), \quad \mathcal{P}_{1}=\bigoplus_{j=1}^{l} \mathcal{O}\left(n_{j}\right), \quad m_{i}, n_{j} \in[0, b),
$$

and let $E^{ \pm}:=\eta_{\mathcal{U}}^{ \pm *} E \in D_{\mathbb{C}^{*}}\left(Y_{\mathcal{U}}^{ \pm}, w^{\star}\right)$, where $\eta^{ \pm}: Y^{ \pm} \hookrightarrow\left[\left(V^{+} \times V^{-}\right) / \mathbb{C}^{*}\right]$ are the natural open immersions. By definition, we have $\Psi^{\prime}\left(E^{-}\right)=E^{+}$. We need to construct a functorial isomorphism

$$
\theta_{E}: E^{+} \stackrel{\cong}{\rightarrow} \Phi\left(E^{-}\right) \text {. }
$$

For this, it is enough to prove Lemma 5.5 below.

Lemma 5.5. Take integers $m, n \in[0, b)$ and $a \mathbb{C}^{*}$-equivariant morphism $f: \mathcal{O}(m) \rightarrow \mathcal{O}(n)$ on $\left(V^{+} \times V^{-}\right) \mathcal{U}$. Put $f^{ \pm}:=\eta_{\mathcal{U}}^{ \pm *}(f): \mathcal{O}_{Y_{\mathcal{U}}^{ \pm}}( \pm m) \rightarrow$ $\mathcal{O}_{Y_{\mathcal{U}}^{ \pm}}( \pm n)$. Then there exist isomorphisms $\theta_{i}: \mathcal{O}_{Y_{\mathcal{U}}^{+}}(i) \rightarrow \Phi\left(\mathcal{O}_{Y_{\mathcal{U}}^{-}}(-i)\right)$ for $i=m, n$, fitting into the commutative diagram

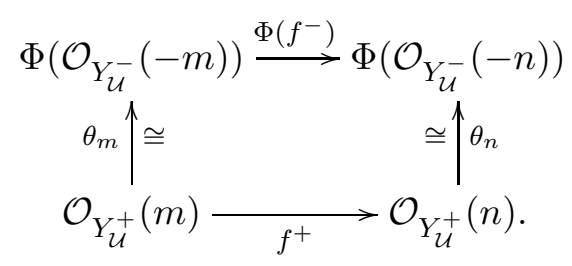


Proof. We first recall the descriptions of $W$ and the morphisms $q^{ \pm}: W \rightarrow$ $Y^{ \pm}$in terms of GIT quotients. We have

$$
W=\left[\left(V^{+*} \times V^{-*} \times \mathbb{C}\right) /\left(\mathbb{C}^{*}\right)^{2}\right],
$$

where $\left(s_{1}, s_{2}\right) \in\left(\mathbb{C}^{*}\right)^{2}$ acts on $V^{+} \times V^{-} \times \mathbb{C}$ as

$$
\left(s_{1}, s_{2}\right) \cdot(\vec{x}, \vec{y}, t):=\left(s_{1} \vec{x}, s_{2}^{-1} \vec{y}, s_{1}^{-1} s_{2} t\right),
$$

and the morphisms $q^{ \pm}: W \rightarrow Y^{ \pm}$are induced by the homomorphisms

$$
\zeta^{ \pm}: V^{+} \times V^{-} \times \mathbb{C} \rightarrow V^{+} \times V^{-}, \quad\left(\mathbb{C}^{*}\right)^{2} \rightarrow \mathbb{C}^{*}
$$

defined by

$$
\begin{array}{rlrl}
\zeta^{+}:(\vec{x}, \vec{y}, t) & \mapsto(\vec{x}, t \vec{y}), & \left(s_{1}, s_{2}\right) & \mapsto s_{1}, \\
\zeta^{-}:(\vec{x}, \vec{y}, t) & \mapsto(t \vec{x}, \vec{y}), & \left(s_{1}, s_{2}\right) \mapsto s_{2},
\end{array}
$$

respectively.

By the descriptions of the morphisms $\zeta^{ \pm}$, we have the following commutative diagram:

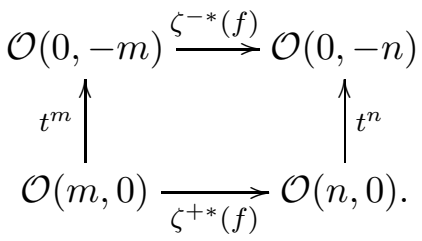

Here, $\mathcal{O}(i, j)$ denotes the $\left(\mathbb{C}^{*}\right)^{2}$-equivariant trivial line bundle on $\mathcal{U} \times V^{+} \times$ $V^{-} \times \mathbb{C}$ of weight $(i, j) \in \mathbb{Z}^{2}$. Pulling back the diagram (5.6) to $W_{\mathcal{U}}$, we get

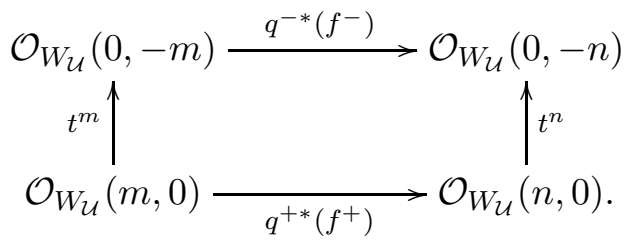

In the above diagram, the vertical morphisms are induced by the morphism

$$
\mathcal{O}_{W_{\mathcal{U}}} \stackrel{t^{d}}{\rightarrow} \mathcal{O}_{W_{\mathcal{U}}}(-d,-d)=\mathcal{O}_{W_{\mathcal{U}}}(d D),
$$

where $d \in \mathbb{Z}$ is an integer and $D \subset W_{\mathcal{U}}$ is the exceptional divisor of $q^{ \pm}$. Furthermore, if $d \in[0, b)$, then the morphism $\mathbf{R} q_{*}^{+}\left(t^{d}\right): \mathcal{O}_{Y_{\mathcal{U}}^{+}} \rightarrow \mathbf{R} p_{*}^{+} \mathcal{O}_{W_{\mathcal{U}}}(d D)$ is an isomorphism. Since we assume $m, n \in[0, b)$, by applying the functor $\mathbf{R} q_{*}^{+}$to the diagram (5.7), we get the commutative diagram as in (5.5).

Let us consider the d-critical loci

$$
M_{\left(Q^{\dagger}, w\right), \mathcal{U}}^{ \pm}=\left\{d w^{\star}=0\right\} \subset Y_{\mathcal{U}}^{ \pm}
$$


By the diagram (4.24) together with Proposition 4.7, the above d-critical loci are smooth and contained in $\mathbb{P}\left(Y^{ \pm}\right)_{\mathcal{U}}$. Explicitly, using the functions $w_{i j}(\vec{u})$ in (5.3), we have

$$
\begin{aligned}
& M_{\left(Q^{\dagger}, w\right), \mathcal{U}}^{+}=\left\{(\vec{x}, \vec{u}) \in \mathbb{P}\left(V^{+}\right)_{\mathcal{U}}: \sum_{i=1}^{a} x_{i} w_{i j}(\vec{u})=0 \text { for all } 1 \leq j \leq b\right\}, \\
& M_{\left(Q^{\dagger}, w\right), \mathcal{U}}^{-}=\left\{(\vec{y}, \vec{u}) \in \mathbb{P}\left(V^{+}\right)_{\mathcal{U}}: \sum_{j=1}^{b} x_{i} w_{i j}(\vec{u})=0 \text { for all } 1 \leq i \leq a\right\} .
\end{aligned}
$$

Let us consider the following diagram

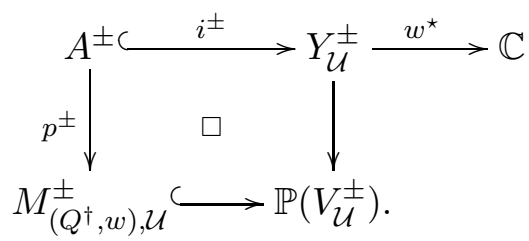

By Theorem 2.3, we have the equivalences

$$
i_{*}^{ \pm} p^{ \pm *}: D^{b}\left(M_{\left(Q^{\dagger}, w\right), \mathcal{U}}^{ \pm} \stackrel{\sim}{\rightarrow} D_{\mathbb{C}^{*}}\left(Y_{\mathcal{U}}^{ \pm}, w^{\star}\right) .\right.
$$

Now let us consider the functor $\Theta_{M}$ defined by the following commutative diagram.

$$
\begin{array}{r}
D^{b}\left(M_{\left(Q^{\dagger}, w\right), \mathcal{U}}^{-}\right) \stackrel{\Theta_{M}}{\longrightarrow} D^{b}\left(M_{\left(Q^{\dagger}, w\right), \mathcal{U}}^{+}\right) \\
i_{*}^{-} p^{-*} \unrhd \cong \\
D_{\mathbb{C}^{*}}\left(Y_{\mathcal{U}}^{-}, w^{\star}\right) \underset{i_{*}^{+} p^{+*}}{\stackrel{\Psi}{\longrightarrow}} D_{\mathbb{C}^{*}}\left(Y_{\mathcal{U}}^{+}, w^{\star}\right) .
\end{array}
$$

To determine the kernel of the functor $\Theta_{M}$, we recall the results from [Todb]. Let $B \subset\left(\mathbb{P}\left(V^{+}\right) \times \mathbb{P}\left(V^{-}\right)\right)_{\mathcal{U}}$ be the subvariety defined by

$$
B:=\left\{(\vec{x}, \vec{y}, \vec{u}) \in\left(\mathbb{P}\left(V^{+}\right) \times \mathbb{P}\left(V^{-}\right)\right)_{\mathcal{U}}: \sum_{i, j} x_{i} y_{j} w_{i j}(\vec{u})=0\right\} .
$$

We have the following diagram:

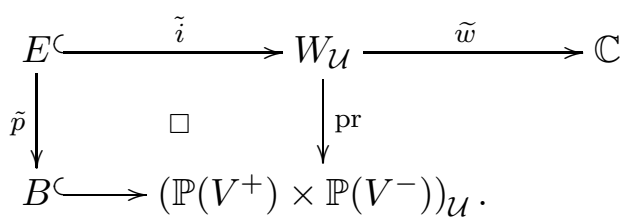


Here $\widetilde{w}$ is the pull-back of $w^{\star}$ by $q^{ \pm}: W_{\mathcal{U}} \rightarrow Y_{\mathcal{U}}^{ \pm}$. We also define subvarieties $F^{ \pm} \subset\left(\mathbb{P}\left(V^{+}\right) \times \mathbb{P}\left(V^{-}\right)\right)_{\mathcal{U}}$ as follows.

$$
\begin{aligned}
& F^{+}:=\left\{(\vec{x}, \vec{y}, \vec{u}) \in\left(\mathbb{P}\left(V^{+}\right) \times \mathbb{P}\left(V^{-}\right)\right)_{\mathcal{U}}: \sum_{i=1}^{a} x_{i} w_{i j}(\vec{u})=0 \text { for all } 1 \leq j \leq b\right\}, \\
& F^{-}:=\left\{(\vec{x}, \vec{y}, \vec{u}) \in\left(\mathbb{P}\left(V^{+}\right) \times \mathbb{P}\left(V^{-}\right)\right)_{\mathcal{U}}: \sum_{j=1}^{b} y_{j} w_{i j}(\vec{u})=0 \text { for all } 1 \leq i \leq a\right\} .
\end{aligned}
$$

We obtain the following diagram:

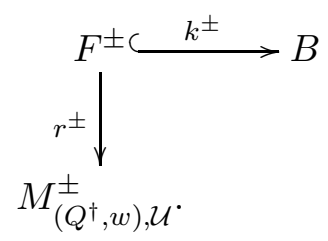

Here $r^{ \pm}$are the projections and $k^{ \pm}$are the closed immersions. Define functors $\Theta^{ \pm}$as

$$
\Theta^{ \pm}:=k_{*}^{ \pm} \circ r^{ \pm *}: D^{b}\left(M_{\left(Q^{\dagger}, w\right), \mathcal{U}}^{ \pm}\right) \rightarrow D^{b}(B),
$$

and let $\Theta_{\mathbf{R}}^{ \pm}:=\mathbf{R} r_{*}^{ \pm} \circ\left(k^{ \pm}\right)^{!}$be the right adjoints of the functors $\Theta^{ \pm}$. We have the following results:

Lemma 5.6 ([Todb, Lemma 3.4, Lemma 3.5]). The following diagram is commutative:

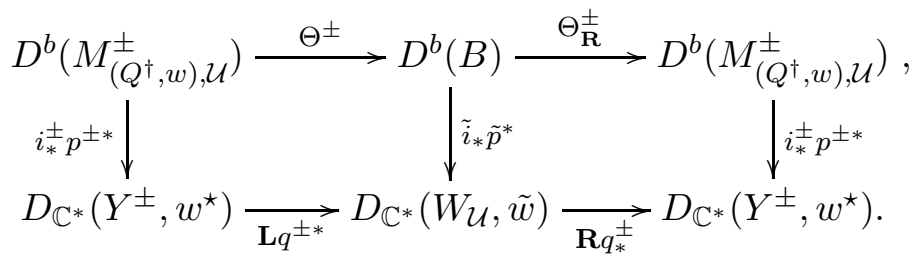

Proposition 5.7 ([Todb, Proposition 3.6]). We have an isomorphism of functors

$$
\Theta_{\mathbf{R}}^{+} \circ \Theta^{-} \cong \otimes \mathcal{O}_{M_{\left(Q^{\dagger}, w\right), \mathcal{U}}^{+}}(b-1) \circ \Phi^{\mathcal{P}} \circ \otimes \mathcal{O}_{M_{\left(Q^{\dagger}, w\right), \mathcal{U}}^{-}}(-1)[1-b],
$$

where we put $\mathcal{P}:=\mathcal{O}_{M_{\left(Q^{\dagger}, w\right), \mathcal{U}}^{-}} \times \mathcal{U}_{\left(_{\left(Q^{\dagger}, w\right), \mathcal{U}}^{+}\right.}$, and $\Phi^{\mathcal{P}}$ is the Fourier-Mukai functor with kernel $\mathcal{P}$.

Let $\pi^{ \pm}: M_{\left(Q^{\dagger}, w\right), \mathcal{U}}^{ \pm} \rightarrow \mathcal{U}$ be the projections. For every integer $i \in \mathbb{Z}$, we define the functor $\bar{\Upsilon}^{i}$ as

$$
\bar{\Upsilon}^{i}:=\otimes \mathcal{O}_{M_{\left(Q^{\dagger}, w\right), \mathcal{U}}^{+}}(i) \circ \mathbf{L} \pi^{+*}: D^{b}(\mathcal{U}) \rightarrow D^{b}\left(M_{\left(Q^{\dagger}, w\right), \mathcal{U}}^{+}\right) .
$$


Lemma 5.8 ([Todb, Lemma 3.7]). For each integer $i \in \mathbb{Z}$, the following diagram is commutative

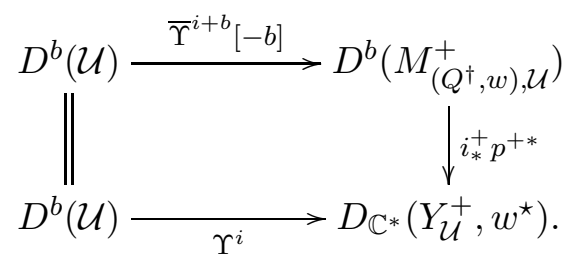

Now we can describe the functor $\Theta_{M}$ explicitly.

Proposition 5.9. We have an isomorphism of functors

$$
\Theta_{M} \cong \otimes \mathcal{O}_{M_{\left(Q^{\dagger}, w\right), \mathcal{U}}^{+}}\left(\left\lfloor\frac{b}{2}\right\rfloor\right) \circ \Phi^{\mathcal{P}} \circ \otimes \mathcal{O}_{M_{\left(Q^{\dagger}, w\right), \mathcal{U}}^{-}}\left(-\left\lceil\frac{b}{2}\right\rceil\right)[1-b] .
$$

Proof. By Proposition 5.4 and the commutativity of the diagrams (5.8), we have

$$
\begin{aligned}
& \Psi \circ i_{*}^{-} p^{-*} \\
& =\otimes \mathcal{O}_{Y_{\mathcal{U}}^{+}}\left(-\left\lceil\frac{b}{2}\right\rceil+1\right) \circ \Phi^{\mathcal{O}_{W_{\mathcal{U}}} \circ \otimes \mathcal{O}_{Y_{\mathcal{U}}^{-}}}\left(-\left\lceil\frac{b}{2}\right\rceil+1\right) \circ i_{*}^{-} p^{-*} \\
& =\otimes \mathcal{O}_{Y_{\mathcal{U}}^{+}}\left(-\left\lceil\frac{b}{2}\right\rceil+1\right) \circ \mathbf{R} q_{*}^{+} \circ \mathbf{L} q^{-*} \circ\left(\mathcal{O}_{Y_{\mathcal{U}}^{-}}\left(-\left\lceil\frac{b}{2}\right\rceil+1\right) \otimes i_{*}^{-} p^{-*}(-)\right) \\
& \cong \otimes \mathcal{O}_{Y_{\mathcal{U}}^{+}}\left(-\left\lceil\frac{b}{2}\right\rceil+1\right) \circ \mathbf{R} q_{*}^{+}\left(\mathcal{O}_{W \mathcal{U}}\left(0,-\left\lceil\frac{b}{2}\right\rceil+1\right) \otimes \mathbf{L} q^{-*} \circ i_{*}^{-} p^{-*}(-)\right) \\
& \cong \otimes \mathcal{O}_{Y_{\mathcal{U}}^{+}}\left(-\left\lceil\frac{b}{2}\right\rceil+1\right) \circ \mathbf{R} q_{*}^{+}\left(\mathcal{O}_{W_{\mathcal{U}}}\left(0,-\left\lceil\frac{b}{2}\right\rceil+1\right) \otimes \tilde{i}_{*} \tilde{p}^{*} \circ \Theta^{-}(-)\right) \\
& \cong \otimes \mathcal{O}_{Y_{\mathcal{U}}^{+}}\left(-\left\lceil\frac{b}{2}\right\rceil+1\right) \circ \mathbf{R} q_{*}^{+} \tilde{i}_{*} \tilde{p}^{*}\left(\mathcal{O}_{B}\left(0,-\left\lceil\frac{b}{2}\right\rceil+1\right) \otimes \Theta^{-}(-)\right) \\
& \cong \otimes \mathcal{O}_{Y_{\mathcal{U}}^{+}}\left(-\left\lceil\frac{b}{2}\right\rceil+1\right) \circ i_{*}^{+} p^{+*} \circ \Theta_{\mathbf{R}}^{+} \circ \Theta^{-} \circ \otimes \mathcal{O}_{M_{\left(Q^{\dagger}, w\right), \mathcal{U}}^{-}}\left(-\left\lceil\frac{b}{2}\right\rceil+1\right) \\
& \cong i_{*}^{+} p^{+*} \circ\left(\otimes \mathcal{O}_{M_{\left(Q^{\dagger}, w\right), \mathcal{U}}^{-}}\left(-\left\lceil\frac{b}{2}\right\rceil+1\right) \circ \Theta_{\mathbf{R}}^{+} \circ \Theta^{-} \circ \otimes \mathcal{O}_{M_{\left(Q^{\dagger}, w\right), \mathcal{U}}^{-}}\left(-\left\lceil\frac{b}{2}\right\rceil+1\right)\right) .
\end{aligned}
$$

Hence the assertion follows from the equation (5.9).

5.4. Descriptions of the kernel object: global case. Denote by

$$
\Phi_{M}: D^{b}\left(M^{\mathrm{DT}}(r, d)\right) \hookrightarrow D^{b}\left(M^{T}(r, d)\right)
$$

the fully faithful functor given in Theorem 1.1. We consider the base change of the diagram (4.11) to the stable locus $M^{\text {st }}(r, d) \subset M(r, d)$ :

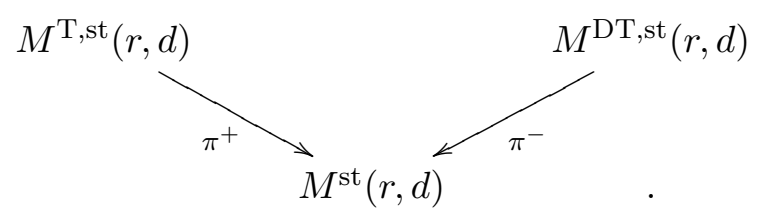


By the construction of $\Phi_{M}$, it restricts to the fully-faithful functor

$$
\Phi_{M^{\mathrm{st}}}: D^{b}\left(M^{\mathrm{DT}, \mathrm{st}}(r, d)\right) \hookrightarrow D^{b}\left(M^{T, \mathrm{st}}(r, d)\right) .
$$

Let $W^{\text {st }}$ be the fiber product

$$
W^{\mathrm{st}}:=M^{\mathrm{T}, \mathrm{st}}(r, d) \times{ }_{M^{\mathrm{st}}(r, d)} M^{\mathrm{DT}, \mathrm{st}}(r, d) .
$$

Suppose that there exists a universal bundle on $C \times M^{\text {st }}(r, d)$, or equivalently

$$
\iota_{M}^{-1}\left(M^{\mathrm{st}}(r, d)\right)=M^{\mathrm{st}}(r, d) \times B \mathbb{C}^{*}
$$

where $\iota_{M}$ is the map (4.6). For an integer $i \in \mathbb{Z}$, let $\mathcal{O}(i)$ be the line bundle on $B \mathbb{C}^{*}$ given by a one dimensional $\mathbb{C}^{*}$-representation with weight $i$, and denote by $\mathcal{O}_{M^{\mathrm{T}, \mathrm{st}}(r, d)}(i)$ its pull-back to $M^{\mathrm{T}, \mathrm{st}}(r, d)$ by the map in the diagram (4.11). We define $\Upsilon_{M^{\text {st }}}^{i}$ to be the functor

$$
\Upsilon_{M^{\text {st }}}^{i}:=\otimes \mathcal{O}_{M^{\mathrm{T}, \mathrm{st}}(r, d)}(i) \circ \mathbf{L} \pi^{+*}: D^{b}\left(M^{\mathrm{st}}(r, d)\right) \rightarrow D^{b}\left(M^{\mathrm{T}, \mathrm{st}}(r, d)\right) .
$$

As a summary of the discussions in this subsection, we have the following result.

Theorem 5.10. The following statements hold.

(i) There exists a line bundle $L$ on $W^{\text {st }}$ and an isomorphism of functors

$$
\Phi_{M^{\mathrm{st}}} \cong \Phi^{i_{W *} L}[1-b],
$$

where $i_{W}: W^{\mathrm{st}} \hookrightarrow M^{\mathrm{T}, \mathrm{st}}(r, d) \times M^{\mathrm{DT}, \mathrm{st}}(r, d)$ is the inclusion.

(ii) For each integer $i \in \mathbb{Z}$, the functor

$$
\Upsilon_{M^{\mathrm{st}}}^{i}: D^{b}\left(M^{\mathrm{st}}(r, d)\right) \rightarrow D^{b}\left(M^{\mathrm{T}, \mathrm{st}}(r, d)\right)
$$

is fully faithful.

(iii) We have the semi-orthogonal decomposition

$$
D^{b}\left(M^{\mathrm{T}, \mathrm{st}}(r, d)\right)=\left\langle\Upsilon_{M^{\mathrm{st}}}^{-\left\lfloor\frac{a}{2}\right\rfloor+b}, \cdots, \Upsilon_{M^{\mathrm{st}}}^{\left.\frac{b}{2}\right\rceil-1}, \Phi_{M^{\mathrm{st}}}, \Upsilon_{M^{\mathrm{st}}}^{\left\lceil\frac{b}{2}\right\rceil}, \cdots, \Upsilon_{M^{\mathrm{st}}}^{\left\lceil\frac{a}{2}\right\rceil-1}\right\rangle
$$

Before the proof, we need a preparation. Let $Y$ be a smooth quasiprojective variety, $X_{i}$ be smooth varieties with projective morphisms $X_{i} \rightarrow$ $Y(i=1,2,3)$. For objects $\mathcal{E} \in D^{b}\left(X_{1} \times X_{2}\right), \mathcal{F} \in D^{b}\left(X_{2} \times X_{3}\right)$, supported on the fiber products $X_{1} \times_{Y} X_{2}, X_{2} \times_{Y} X_{3}$, we define

$$
\mathcal{F} \circ \mathcal{E}:=\mathbf{R} p_{13 *}\left(p_{12}^{*} \mathcal{E} \otimes^{\mathbf{L}} p_{23}^{*} \mathcal{F}\right) \in D^{b}\left(X_{1} \times X_{3}\right) .
$$

Here, $p_{i j}: X_{1} \times X_{2} \times X_{3} \rightarrow X_{i} \times X_{j}$ denotes the projections $(i, j=1,2,3)$. Then we have an isomorphism of functors

$$
\Phi^{\mathcal{F}} \circ \Phi^{\mathcal{E}} \cong \Phi^{\mathcal{F} \circ \mathcal{E}}: D^{b}\left(X_{1}\right) \rightarrow D^{b}\left(X_{3}\right) .
$$

Lemma 5.11. Let $Y$ be a smooth quasi-projective variety, $X_{1}, X_{2}$ smooth varieties, projective over $Y$. Let $\mathcal{E}, \mathcal{F} \in D^{b}\left(X_{1} \times X_{2}\right)$ be objects supported on the fiber product $X_{1} \times_{Y} X_{2}$. Assume that we have an isomorphism of functors

$$
\Phi^{\mathcal{E}} \cong \Phi^{\mathcal{F}}: D^{b}\left(X_{1}\right) \hookrightarrow D^{b}\left(X_{2}\right)
$$


and that they are fully faithful functors. Then we have an isomorphism $\mathcal{E} \cong \mathcal{F}$.

Proof. A similar statement was proved in Tod15, Lemma 4.1] when the functors are equivalences. In particular, by loc. cit., we have an isomorphism

$$
\mathcal{F}^{*} \circ \mathcal{E} \cong \mathcal{O}_{\Delta_{X_{1}}}
$$

Here, the object $\mathcal{F}^{*} \in D^{b}\left(X_{1} \times X_{2}\right)$ is defined as

$$
\mathcal{F}^{*}:=\mathbf{R} \mathcal{H o m}_{X_{1} \times X_{2}}\left(\mathcal{F}, \mathcal{O}_{X_{1} \times X_{2}}\right) \otimes p_{1}^{*} \omega_{X_{1}}\left[\operatorname{dim} X_{1}\right],
$$

where $p_{1}: X_{1} \times X_{2} \rightarrow X_{1}$ is the projection.

On the other hand, there exists an object $\mathcal{G} \in D^{b}\left(X_{2} \times X_{2}\right)$ and an exact triangle

$$
\mathcal{F} \circ \mathcal{F}^{*} \rightarrow \mathcal{O}_{\Delta_{X_{2}}} \rightarrow \mathcal{G}
$$

which induces the adjoint counit $\Phi^{\mathcal{F}} \circ \Phi^{\mathcal{F}^{*}} \rightarrow \operatorname{Id}_{\mathrm{D}^{\mathrm{b}}\left(\mathrm{X}_{2}\right)}$ (cf. AL12, Theorem 3.1]). By the construction, the functor $\Phi^{\mathcal{G}}: D^{b}\left(X_{2}\right) \rightarrow D^{b}\left(X_{2}\right)$ coincides with the projection functor $D^{b}\left(X_{2}\right) \rightarrow\left(\Phi^{\mathcal{F}}\left(D^{b}\left(X_{1}\right)\right)\right)^{\perp}$ to the right orthogonal complement. In particular, we have

$$
\Phi^{\mathcal{G} \circ \mathcal{E}} \cong \Phi^{\mathcal{U}} \circ \Phi^{\mathcal{E}}=0: D^{b}\left(X_{1}\right) \rightarrow\left(\Phi^{\mathcal{F}}\left(D^{b}\left(X_{1}\right)\right)\right)^{\perp}
$$

and hence $\mathcal{G} \circ \mathcal{E} \cong 0$. Applying $(-) \circ \mathcal{E}$ to the exact triangle (5.12), we get the exact triangle

$$
\mathcal{F} \cong \mathcal{F} \circ \mathcal{F}^{*} \circ \mathcal{E} \rightarrow \mathcal{E} \rightarrow \mathcal{G} \circ \mathcal{E} \cong 0
$$

in $D^{b}\left(X_{1} \times X_{2}\right)$, i.e., $\mathcal{F} \cong \mathcal{E}$.

Proof of Theorem 5.10. The second and third statements hold by its analytic local version Theorem 5.3 (see the argument of [Todb, Theorem 4.5]). We prove the first statement. To simplify the notation, we set

$$
M:=M^{\mathrm{st}}(r, d), M^{+}:=M^{\mathrm{T}, \mathrm{st}}(r, d), M^{-}:=M^{\mathrm{DT}, \mathrm{st}}(r, d), W:=W^{\mathrm{st}} .
$$

Denote by $\rho: W \rightarrow M$ the projection. Let $\mathcal{E} \in D^{b}\left(M^{-} \times M^{+}\right)$be the kernel object of the functor $\Phi_{M \mathrm{st}}[b-1]$. By Proposition 5.9 , there exist an analytic open covering $\left\{\mathcal{U}_{i}\right\}_{i \in \mathbb{Z}}$ of $M$ and line bundles $L_{i}$ on $\rho^{-1}\left(\mathcal{U}_{i}\right)$ satisfying the following property: For each $i \in \mathbb{Z}$, we have the commutative diagram

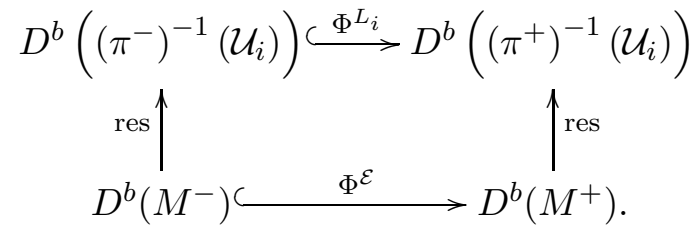

From this, we can see that the object $\mathcal{E}$ is supported on $W$, and we have an isomorphism of functors

$$
\Phi^{\mathcal{E}_{i}} \cong \Phi^{L_{i}}: D^{b}\left(\left(\pi^{-}\right)^{-1}\left(\mathcal{U}_{i}\right)\right) \rightarrow D^{b}\left(\left(\pi^{-}\right)^{-1}\left(\mathcal{U}_{i}\right)\right)
$$


for each $i \in \mathbb{Z}$, where we put $\mathcal{E}_{i}:=\left.\mathcal{E}\right|_{\rho^{-1}\left(\mathcal{U}_{i}\right)}$. Hence by Lemma 5.11, we conclude that the object $\mathcal{E}$ is isomorphic to some line bundle $L$ on $W$.

\section{Comparison with ADHM sheaves}

In this section, we will explain about the hidden Calabi-Yau three structure behind the d-critical structure on the moduli spaces of Thaddeus pairs constructed in Theorem 4.10 .

6.1. ADHM sheaves. In this subsection, we recall the definition and the basic facts about ADHM sheaves studied in [Dia12b, Dia12a, CDP10. Let $C$ be a smooth projective curve of genus $g$, and fix line bundles $M_{1}, M_{2}$ on $C$ such that $M_{1} \otimes M_{2} \cong \omega_{C}^{\vee}$. First we define the notion of Higgs bundles.

Definition 6.1. $\quad$ (i) A Higgs bundle is a triplet $\left(E, \Phi_{1}, \Phi_{2}\right)$, where $E$ is a locally free sheaf on $C$, and

$$
\Phi_{i}: E \otimes M_{i} \rightarrow E,
$$

are morphisms of coherent sheaves satisfying the relation

$$
\Phi_{1} \circ\left(\Phi_{2} \otimes i d_{M_{1}}\right)-\Phi_{2} \circ\left(\Phi_{1} \otimes i d_{M_{2}}\right)=0 .
$$

(ii) Let $\left(E, \Phi_{1}, \Phi_{2}\right)$ be a Higgs bundle. A subsheaf $F \subset E$ is called $\Phi$-invariant if for $i=1,2$, we have $\Phi_{i}\left(F \otimes M_{i}\right) \subset F$.

(iii) A Higgs bundle $\left(E, \Phi_{1}, \Phi_{2}\right)$ is called semistable if for every $\Phi$-invariant subsheaf $F \subset E$, we have $\mu(F) \leq \mu(E)$.

We denote by $H(r, d)$ the good moduli space of semistable Higgs bundles $\left(E, \Phi_{1}, \Phi_{2}\right)$ satisfying the condition

$$
(\operatorname{rk}(E), \chi(E))=(r, d) .
$$

Similarly as in Definition 4.2, we define the following notions:

Definition 6.2. $\quad$ (i) A semistable ADHM sheaf is a quadruplet

$$
\left(E, \Phi_{1}, \Phi_{2}, \psi\right)
$$

consisting of a semistable Higgs bundle $\left(E, \Phi_{1}, \Phi_{2}\right)$ and a morphism $\psi: \mathcal{O}_{C} \rightarrow E$ such that there is no non-zero subbundle $F \subsetneq E$ with $\mu(F)=\mu(E)$ and $\psi\left(\mathcal{O}_{C}\right) \subset F$.

(ii) A semistable dual ADHM sheaf is a quadruplet $\left(E^{\prime}, \Phi_{1}^{\prime}, \Phi_{2}^{\prime}, \psi^{\prime}\right)$ consisting of a semistable Higgs bundle $\left(E^{\prime}, \Phi_{1}^{\prime}, \Phi_{2}^{\prime}\right)$ and a morphism $\psi^{\prime}: E \rightarrow \omega_{C}$ such that there is no non-zero subbundle $F^{\prime} \subsetneq E^{\prime}$ with $\mu(F)=\mu(E)$ and $\psi^{\prime}\left(F^{\prime}\right)=0$.

Remark 6.3. In Dia12a, Definition 2.1], the notion of $\delta$-semistable ADHM sheaves are introduced for a real number $\delta \in \mathbb{R}$. Our notion of semistable (resp. dual) ADHM sheaves is equivalent to the notion of $\delta$-semistable $A D H M$ sheaves for $0<\delta \ll 1$ (resp. $-1 \ll \delta<0$ ). 
We denote by $M^{\operatorname{ADHM}}(r, d)$ (resp. $M^{\operatorname{DADHM}}(r, d)$ ) the moduli space of semistable (resp. dual) ADHM sheaves $\left(E, \Phi_{1}, \Phi_{2}, \psi\right)$ with $\left[E, \Phi_{1}, \Phi_{2}\right] \in$ $H(r, d)$.

We have the following diagram of quasi-projective schemes over $\mathbb{C}$ :

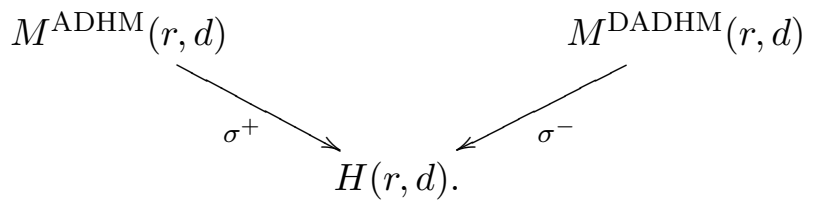

Remark 6.4. Let $X:=\operatorname{Tot}_{C}\left(M_{1}^{\vee} \oplus M_{2}^{\vee}\right)$ be a non-compact Calabi-Yau threefold. It is well-known that giving a Higgs bundle is equivalent to giving a compactly supported one dimensional pure sheaf on $X$. Hence we can think the moduli spaces $M^{\mathrm{ADHM}}(r, d), M^{\mathrm{DADHM}}(r, d)$, and $H(r, d)$ as the moduli spaces of objects in $D^{b}(X)$. In fact, the diagram (6.1) can be described as the wall crossing diagram of the moduli spaces of objects in $D^{b}(X)$, which was studied in [Tod10].

We have the following result.

Theorem 6.5 ([Toda, Theorem 9.13]). For $d>0$, the diagram (6.1) is a $d$-critical flip. For $d=0$, it is a d-critical flop.

6.2. Torus action on ADHM sheaves. In this subsection, we consider the natural $\mathbb{C}^{*}$-action on the moduli space of semistable (dual) ADHM sheaves. We will see that the moduli space of (dual) Thaddeus pairs appear as one of the connected components of $\mathbb{C}^{*}$-fixed locus.

Definition 6.6. We define a $\mathbb{C}^{*}$-action on $M^{\mathrm{ADHM}}(r, d)$ as follows: for $t \in \mathbb{C}^{*}$ and $\left[E, \Phi_{1}, \Phi_{2}, \psi\right] \in M^{\operatorname{ADHM}}(r, d)$,

$$
t \cdot\left(E, \Phi_{1}, \Phi_{2}, \psi\right):=\left(E, t \Phi_{1}, t^{-1} \Phi_{2}, \psi\right) .
$$

We define a $\mathbb{C}^{*}$-action on $M^{\mathrm{DADHM}}(r, d)$ similarly.

We have the following theorem due to Dia12a]:

Theorem 6.7 ([Dia12a, Theorem 1.5]). The $\mathbb{C}^{*}$-fixed loci $M^{\operatorname{ADHM}}(r, d)^{\mathbb{C}^{*}}$ and $M^{\mathrm{DADHM}}(r, d)^{\mathbb{C}^{*}}$ are projective schemes over $\mathbb{C}$.

Now we can prove the following:

Proposition 6.8. The moduli spaces $M^{T}(r, d), M^{\mathrm{DT}}(r, d)$ are one of the connected components of the torus fixed loci $M^{\operatorname{ADHM}}(r, d)^{\mathbb{C}^{*}}, M^{\operatorname{DADHM}}(r, d)^{\mathbb{C}^{*}}$, respectively.

Proof. We only prove the assertion for $M^{T}(r, d)$. By the definitions of stability and the $\mathbb{C}^{*}$-action, we have the inclusion $\phi: M^{T}(r, d) \hookrightarrow M^{\operatorname{ADHM}}(r, d)^{\mathbb{C}^{*}}$. Furthermore, both of these schemes are projective by Theorem 6.7. Hence it is enough to show that the inclusion $\phi$ is an open immersion. 
Take an element

$$
p=I^{\bullet}=\left(\mathcal{O}_{C} \stackrel{s}{\rightarrow} E\right) \in M^{T}(r, d) .
$$

We think $I^{\bullet}$ as an object in the derived category $D^{b}(C)$ so that $\mathcal{O}_{C}$ is located in degree 0. As mentioned in Remark 6.4 the moduli space $M^{\operatorname{ADHM}}(r, d)$ can be described as the moduli space of objects in $D^{b}(X)$. Then we have $J^{\bullet}:=\phi(p)=\left(\mathcal{O}_{X} \stackrel{t}{\rightarrow} \iota_{*} E\right) \in D^{b}(X)$, where $\iota: C \rightarrow X$ is the zero section and the map $t$ is given by the composition

$$
t: \mathcal{O}_{X} \rightarrow \iota_{*} \mathcal{O}_{C} \stackrel{\iota_{*}(s)}{\longrightarrow} \iota_{*} E .
$$

We will show that the tangent map

$$
T_{p} \phi: T_{p} M^{T}(r, d) \rightarrow T_{\phi(p)} M^{\mathrm{ADHM}}(r, d)^{\mathbb{C}^{*}}
$$

is an isomorphism. By the deformation-obstruction theory for pairs, the tangent spaces are given as follows:

$$
\begin{aligned}
& T_{p} M^{T}(r, d)=\operatorname{Hom}_{C}\left(I^{\bullet}, E\right), \\
& T_{\phi(p)} M^{\operatorname{ADHM}}(r, d)^{\mathbb{C}^{*}}=\operatorname{Hom}_{X}\left(J^{\bullet}, \iota_{*} E\right)^{\mathbb{C}^{*}} \cong \operatorname{Hom}_{C}\left(\mathbf{L} \iota^{*} J^{\bullet}, E\right)^{\mathbb{C}^{*}} .
\end{aligned}
$$

Note that by the stability condition, the section $s \in H^{0}(C, E)$ is non-zero, and we have the short exact sequence

$$
0 \rightarrow \mathcal{O}_{C} \rightarrow E \rightarrow Q \rightarrow 0
$$

for some coherent sheaf $Q \in \operatorname{Coh}(C)$. Hence we have

$$
\operatorname{Hom}_{C}\left(I^{\bullet}, E\right) \cong \operatorname{Ext}_{C}^{1}(Q, E) .
$$

To compute the vector space $\operatorname{Hom}_{C}\left(\mathbf{L} \iota^{*} J^{\bullet}, E\right)^{\mathbb{C}^{*}}$, let us apply the functor $\mathbf{L} \iota *$ to the exact triangle

$$
\iota_{*} E[-1] \rightarrow J^{\bullet} \rightarrow \mathcal{O}_{X}
$$

We get the long exact sequence

$$
\begin{gathered}
0 \longrightarrow E \otimes\left(M_{1} \oplus M_{2}\right) \longrightarrow \mathcal{H}^{0}\left(\mathbf{L} \iota^{*} J^{\bullet}\right) \longrightarrow \mathcal{O}_{C} \\
\stackrel{\iota^{*}(t)=s}{\longrightarrow} E \longrightarrow \mathcal{H}^{1}\left(\mathbf{L} \iota^{*} J^{\bullet}\right) \longrightarrow 0
\end{gathered}
$$

Hence we have

$$
\mathcal{H}^{0}\left(\mathbf{L} \iota^{*} J^{\bullet}\right) \cong E \otimes\left(M_{1} \oplus M_{2}\right), \quad \mathcal{H}^{1}\left(\mathbf{L} \iota^{*} J^{\bullet}\right) \cong Q,
$$

and $\mathcal{H}^{i}\left(\mathbf{L} \iota^{*} J^{\bullet}\right)=0$ for $i \geq 2$. Furthermore, by the definition of the $\mathbb{C}^{*}$ action, we have the vanishing $\operatorname{Hom}_{C}\left(E \otimes\left(M_{1} \oplus M_{2}\right), E\right)^{\mathbb{C}^{*}}=0$. We conclude that

$$
\operatorname{Hom}_{C}\left(\mathbf{L} \iota^{*} J^{\bullet}, E\right)^{\mathbb{C}^{*}} \cong \operatorname{Ext}_{C}^{1}(Q, E)
$$

as required. 
Recall that our $\mathbb{C}^{*}$-action on ADHM sheaves are defined so that its weights are $1,-1$ on $M_{1}, M_{2}$, respectively. In particular, the action preserves the isomorphism $M_{1} \otimes M_{2} \cong \omega_{C}^{\vee}$. Hence by taking fixed loci, the relative dcritical charts on the diagram (6.1) induce the relative d-critical charts on the wall crossing diagram of moduli spaces of Thaddeus pairs considered in Theorem 4.10.

\section{Appendix A. Review on D-CRitical Birational geOmetry}

Here we recall the basic notions in d-critical birational geometry introduced by the second author [Toda, Todb].

A.1. D-critical loci. In this subsection, we quickly review about the notion of (analytic) d-critical loci introduced by Joyce. For more detail, see his original paper Joy15]. Let $M$ be a complex analytic space. Then there exists a sheaf $\mathcal{S}_{M}$ of $\mathbb{C}$-vector spaces on $M$ satisfying the following property: for any analytic open subset $U \subset M$ and a closed immersion $i: U \hookrightarrow Y$ into a smooth complex manifold $Y$, there exists an exact sequence

$$
\left.0 \rightarrow \mathcal{S}_{M}\right|_{U} \rightarrow \mathcal{O}_{Y} / I^{2} \rightarrow \Omega_{Y} / I^{2}
$$

where $I \subset \mathcal{O}_{Y}$ is the ideal sheaf defining $U \subset Y$. Furthermore, there exists a direct sum decomposition

$$
\mathcal{S}_{M}=\mathbb{C}_{M} \oplus \mathcal{S}_{M}^{0}
$$

The following is the basic example.

Example A.1. Let $Y$ be a complex manifold, $U \subset Y$ a closed analytic subspace. Assume that there exists a holomorphic function $w: Y \rightarrow \mathbb{C}$ satisfying

$$
U=\{d w=0\} \subset Y,\left.\quad w\right|_{U^{\text {red }}}=0 .
$$

Then the section

$$
s:=w+I^{2} \in H^{0}\left(U, \mathcal{O}_{Y} / I^{2}\right)
$$

is in fact an element of $H^{0}\left(U, \mathcal{S}_{U}^{0}\right)$. Here, $I=(d w) \subset \mathcal{O}_{Y}$ is the ideal sheaf defining $U \subset Y$.

Definition A.2. A d-critical locus is a pair $(M, s)$ consisting of a complex analytic space $M$ and a section $s \in H^{0}\left(M, \mathcal{S}_{M}^{0}\right)$ satisfying the following property: for every point $x \in M$, there exist an open neighborhood $x \in U \subset$ $M$ of $x$, a closed embedding $i: U \hookrightarrow Y$ into a complex manifold $Y$, and a holomorphic function $w: Y \rightarrow \mathbb{C}$ satisfying the conditions (A.1) such that the restriction $\left.s\right|_{U}$ can be written as (A.2) in Example A.1. We call the data $(U, Y, w, i)$ as a d-critical chart, and the section $s$ as a d-critical structure.

The following definition is a relative version of d-critical charts. 
Definition A.3. Let $(M, s)$ be a d-critical locus, $\pi: M \rightarrow A$ a morphism of analytic spaces. Let $U \subset A$ be an open subset. Assume that there exists a following commutative diagram

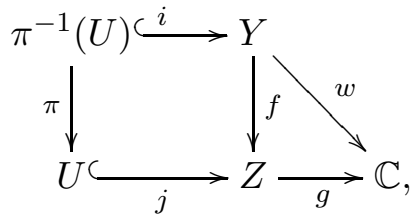

where $f$ is a morphism of analytic spaces with $Y$ smooth, $i, j$ are closed embeddings, and $g$ is a holomorphic function. If the data $\left(\pi^{-1}(U), Y, w, i\right)$ defines a d-critical chart of $(M, s)$, then we call it as a $\pi$-relative $d$-critical chart.

A.2. D-critical birational transforms. We define the d-critical analogue of birational transforms. For the standard terminologies in birational geometry, we refer to [KMM87, KM98]. See also [Toda, Section 2].

Definition A.4. Let $\left(M^{ \pm}, s^{ \pm}\right)$be d-critical loci, $\pi^{ \pm}: M^{ \pm} \rightarrow A$ be morphisms of analytic spaces. Then the diagram

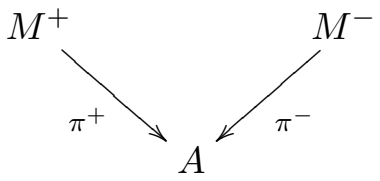

is called a d-critical flip, $d$-critical flop, at a point $p \in A$ if there exist an open neighborhood $p \in U \subset A$ and $\pi^{ \pm}$-relative $d$-critical charts

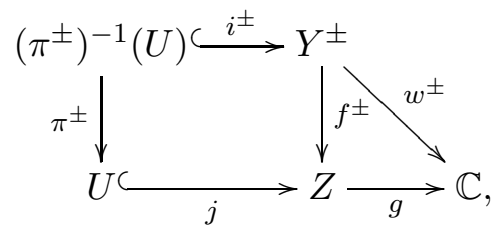

with $g$ and $j$ are independent on \pm , such that the diagram

$$
Y^{+} \stackrel{f^{+}}{\longrightarrow} Z \stackrel{f^{-}}{\longleftarrow} Y^{-}
$$

is a (usual) flip, flop, respectively.

We call the digram (A.3) as a d-critical d-critical flip, d-critical flop, if it satisfies the corresponding condition at any point $p \in A$.

We can see that d-critical birational transforms defined above decrease virtual canonical line bundles on d-critical loci. See [Toda, Section 3] for more detail. 


\section{REFERENCES}

[AL12] Rina Anno and Timothy Logvinenko, On adjunctions for Fourier-Mukai transforms, Adv. Math. 231 (2012), no. 3-4, 2069-2115. MR 2964634

[Bal17] Matthew Robert Ballard, Wall crossing for derived categories of moduli spaces of sheaves on rational surfaces, Algebr. Geom. 4 (2017), no. 3, 263-280. MR 3652079

[BFK14] Matthew Ballard, David Favero, and Ludmil Katzarkov, A category of kernels for equivariant factorizations and its implications for Hodge theory, Publ. Math. Inst. Hautes Études Sci. 120 (2014), 1-111. MR 3270588

[BFK19] Variation of geometric invariant theory quotients and derived categories, J. Reine Angew. Math. 746 (2019), 235-303. MR 3895631

[BO] A. Bondal and D. Orlov, Semiorthogonal decomposition for algebraic varieties, preprint, arXiv:9506012.

[CDP10] W. Y. Chuang, D. E. Diaconescu, and G. Pan, Rank two ADHM invariants and wallcrossing, Commun. Number Theory Phys. 4 (2010), 417-461.

[CIJS15] Tom Coates, Hiroshi Iritani, Yunfeng Jiang, and Ed Segal, K-theoretic and categorical properties of toric Deligne-Mumford stacks, Pure Appl. Math. Q. 11 (2015), no. 2, 239-266. MR 3544765

[Dia12a] D.-E. Diaconescu, Chamber structure and wallcrossing in the ADHM theory of curves, I, J. Geom. Phys. 62 (2012), no. 2, 523-547. MR 2864495

[Dia12b] Moduli of ADHM sheaves and the local Donaldson-Thomas theory, J. Geom. Phys. 62 (2012), no. 4, 763-799. MR 2888981

[EP15] Alexander I. Efimov and Leonid Positselski, Coherent analogues of matrix factorizations and relative singularity categories, Algebra Number Theory 9 (2015), no. 5, 1159-1292. MR 3366002

[Hir17] Yuki Hirano, Derived Knörrer periodicity and Orlov's theorem for gauged Landau-Ginzburg models, Compos. Math. 153 (2017), no. 5, 973-1007. MR 3631231

[HLa] D. Halpern-Leistner, The D-equivalence conjecture for moduli spaces of sheaves on a K3 surface, available in http://www.math.columbia.edu/ danhl/.

[HLb] - On the structure of instability in moduli theory, arXiv:1411.0627.

[HLc] Remarks on Theta-stratifications and derived categories, arXiv:1502.03083.

[HL97] Daniel Huybrechts and Manfred Lehn, The geometry of moduli spaces of sheaves, Aspects of Mathematics, E31, Friedr. Vieweg \& Sohn, Braunschweig, 1997. MR 1450870

[HL15] Daniel Halpern-Leistner, The derived category of a GIT quotient, J. Amer. Math. Soc. 28 (2015), no. 3, 871-912. MR 3327537

[HLS] D. Halpern-Leistner and S. V. Sam, Combinatorial constructions of derived equivalences, arXiv:1601.02030.

[Isi13] Mehmet Umut Isik, Equivalence of the derived category of a variety with a singularity category, Int. Math. Res. Not. IMRN (2013), no. 12, 2787-2808. MR 3071664

[Joy15] D. Joyce, A classical model for derived critical loci, J. Differential Geom. 101 (2015), 289-367.

[Kaw02] Y. Kawamata, D-equivalence and K-equivalence, J. Differential Geom. 61 (2002), 147-171.

[Kin94] A. King, Moduli of representations of finite-dimensional algebras, Quart. J. Math. Oxford Ser.(2) 45 (1994), 515-530.

[KM98] J. Kollár and S. Mori, Birational geometry of algebraic varieties, Cambridge Tracts in Mathematics, vol. 134, Cambridge University Press, 1998. 
[KMM87] Y. Kawamata, K. Matsuda, and K. Matsuki, Introduction to the Minimal Model Problem, Adv. Stud. Pure Math 10 (1987), 283-360.

[Lin18] Yinbang Lin, Moduli spaces of stable pairs, Pacific J. Math. 294 (2018), no. 1, 123-158. MR 3743369

[Pot16] N. Potashnik, Derived Categories of Moduli Spaces of Semistable Pairs over Curves, PhD thesis, Columbia University (2016).

[PT09] R. Pandharipande and R. P. Thomas, Curve counting via stable pairs in the derived category, Invent. Math. 178 (2009), 407-447.

[Shi12] Ian Shipman, A geometric approach to Orlov's theorem, Compos. Math. 148 (2012), no. 5, 1365-1389. MR 2982435

[ST11] Jacopo Stoppa and Richard P. Thomas, Hilbert schemes and stable pairs: GIT and derived category wall crossings, Bull. Soc. Math. France 139 (2011), no. 3, 297-339. MR 2869309

[Tha94] Michael Thaddeus, Stable pairs, linear systems and the Verlinde formula, Invent. Math. 117 (1994), no. 2, 317-353. MR 1273268

[Toda] Y. Toda, Birational geometry for d-critical loci and wall-crossing in Calabi-Yau 3-folds, arXiv:1805.00182.

[Todb] Semiorthogonal decompositions of stable pair moduli spaces via dcritical flips, arXiv:1805.00183.

[Tod10] _ Generating functions of stable pair invariants via wall-crossings in derived categories, Adv. Stud. Pure Math. 59 (2010), 389-434, New developments in algebraic geometry, integrable systems and mirror symmetry (RIMS, Kyoto, 2008).

[Tod12] Stability conditions and curve counting invariants on Calabi-Yau 3folds, Kyoto Journal of Mathematics 52 (2012), 1-50.

[Tod14] Yukinobu Toda, Multiple cover formula of generalized DT invariants I: Parabolic stable pairs, Adv. Math. 257 (2014), 476-526. MR 3187656

[Tod15] Non-commutative width and Gopakumar-Vafa invariants, Manuscripta Math. 148 (2015), no. 3-4, 521-533. MR 3414491

[Tod18] Moduli stacks of semistable sheaves and representations of Ext-quivers, Geom. Topol. 22 (2018), no. 5, 3083-3144. MR 3811778

[vVdB17] Špela Špenko and Michel Van den Bergh, Non-commutative resolutions of quotient singularities for reductive groups, Invent. Math. 210 (2017), no. 1, 3-67. MR 3698338

Graduate School of Mathematical Sciences, The University of Tokyo 3-8-1 Komaba, Meguro, TOKYo, 153-8914 Japan

E-mail address: koseki@ms.u-tokyo.ac.jp

Kavli Institute for the Physics and Mathematics of the Universe (WPI), The University of Tokyo Institutes for Advanced Study, The University of TOKyo, KASHIWA, CHIBA 277-8583, JAPAN

E-mail address: yukinobu.toda@ipmu.jp 